\title{
Hybrid-Game Strategies for multi-objective design optimization in engineering
}

\author{
DongSeop Lee ${ }^{\mathrm{a}, \mathrm{b}, *}$, Luis Felipe Gonzalez ${ }^{\mathrm{c}, 1}$, Jacques Periaux ${ }^{\mathrm{a}, \mathrm{d}, 2}$, Karkenahalli Srinivas ${ }^{\mathrm{b}, 3}$, Eugenio Onate ${ }^{\mathrm{a}, \mathrm{d}, 4}$ \\ ${ }^{a}$ International Center for Numerical Methods in Engineering, (CIMNE), Edificio C1, 08034 Barcelona, Spain \\ ${ }^{\mathrm{b}}$ School of Aerospace Mechanical \& Mechatronic Engineering (AMME),University of Sydney, NSW 2006, Australia \\ ${ }^{\mathrm{c}}$ School of Engineering System, Queensland University of Technology, Brisbane, Australia \\ ${ }^{\mathrm{d}}$ Universitat Politecnica de Catalunya (UPC), 08034 Barcelona, Spain
}

\section{A R T I C L E I N F O}

\section{Article history:}

Received 22 December 2009

Received in revised form 17 August 2010

Accepted 10 March 2011

Available online 21 March 2011

\section{Keywords:}

Multi-Objective Evolutionary Algorithm

(MOEA)

Shape optimization

Game Strategies

Hybrid-Game

Nash-equilibrium

Pareto front

\begin{abstract}
A B S T R A C T
A number of Game Strategies (GS) have been developed in past decades. They have been used in the fields of economics, engineering, computer science and biology due to their efficiency in solving design optimization problems. In addition, research in multi-objective (MO) and multidisciplinary design optimization (MDO) has focused on developing robust and efficient optimization methods to produce a set of high quality solutions with low computational cost. In this paper, two optimization techniques are considered; the first optimization method uses multi-fidelity hierarchical Pareto optimality. The second optimization method uses the combination of two Game Strategies; Nash-equilibrium and Pareto optimality. The paper shows how Game Strategies can be hybridised and coupled to Multi-Objective Evolutionary Algorithms (MOEA) to accelerate convergence speed and to produce a set of high quality solutions. Numerical results obtained from both optimization methods are compared in terms of computational expense and model quality. The benefits of using Hybrid-Game Strategies are clearly demonstrated.
\end{abstract}

(C) 2011 Elsevier Ltd. All rights reserved.

\section{Introduction}

With an ever-increasing complexity in design engineering problems, research of multi-objective (MO) and multidisciplinary design optimization (MDO) focuses on developing robust and efficient optimization methods to produce high quality designs with low computational cost [1-4]. In such situations, Game Strategies (GSs) has proposed as one of the key technologies to save CPU usage and produce high model quality due to their efficiency in design optimization [4,5,7]. In this paper, two GSs are considered and applied to two optimization methods; the first optimization method employs the concept of multi-fidelity hierarchical Pareto optimality evolutionary algorithm (HAPMOEA) [6]. The second method uses a combination of the concepts of Nash-equilibrium [7] and Pareto optimality [8] (herein named Hybrid-Game) coupled to Multi-Objective Evolutionary Algorithm (MOEA). HAPMOEA

* Corresponding author at: Parque Mediterraneo de la Tecnologia (PMT), Edificio C3, Room 312, CIMNE/UPC, C/Esteve Terradas n5, 08860 Castelldefels, Spain. Tel.: +34934015 696; fax: +34934016517.

E-mail addresses: ds.chris.lee@gmail.com (D. Lee), felipe.gonzalez@qut.edu.au (L.F. Gonzalez), jperiaux@gmail.com (J. Periaux), k.srinivas@usyd.edu.au (K. Srinivas), onate@cimne.upc.edu (E. Onate).

1 Tel.: +61 73138 1363; fax: +61 731381469 .

2 Tel.: +34932057 016; fax: +34934016517.

3 Tel.: +6129351 4289; fax: +61293514841.

4 Tel.: +34 932057 016; fax: +34934016517. uses three hierarchical layers with seven populations (Paretogames) which are divided by model fidelity conditions. HybridGame consists of one Pareto-Player and several Nash-Players and can produce a Nash-equilibrium and Pareto non-dominated solutions simultaneously [9]. The reason of using Nash-game is to use it as pre-conditioner. It speeds up the search for a local solution which will then be seeded to the Pareto-Player to produce global solutions. The hybrid game is especially developed to solve complex design problems such as robust MO/MDO which requires high computational cost.

The evolutionary optimization methods HAPMOEA and HybridGame are coupled to a Multi-Objective Evolutionary Algorithms (MOEA) in an asynchronous parallel computation and are implemented to solve a single-disciplinary multi-objective design and uncertainty based multidisciplinary design problems.

The rest of paper is organized as followed; Section 2 describes the methodologies and presents algorithms for HAPMOEA and Hybrid-Game. Section 3 presents the aerodynamic analysis tools used in this work. Real world MO design problem is conducted in Section 4. Conclusions are presented in Section 5.

\section{Methodology}

Both methods HAPMOEA and Hybrid-Game have same feature of Multi-Objective Evolutionary Algorithms (MOEAs). HAPMOEA uses hierarchical multi-population Pareto optimality while the 


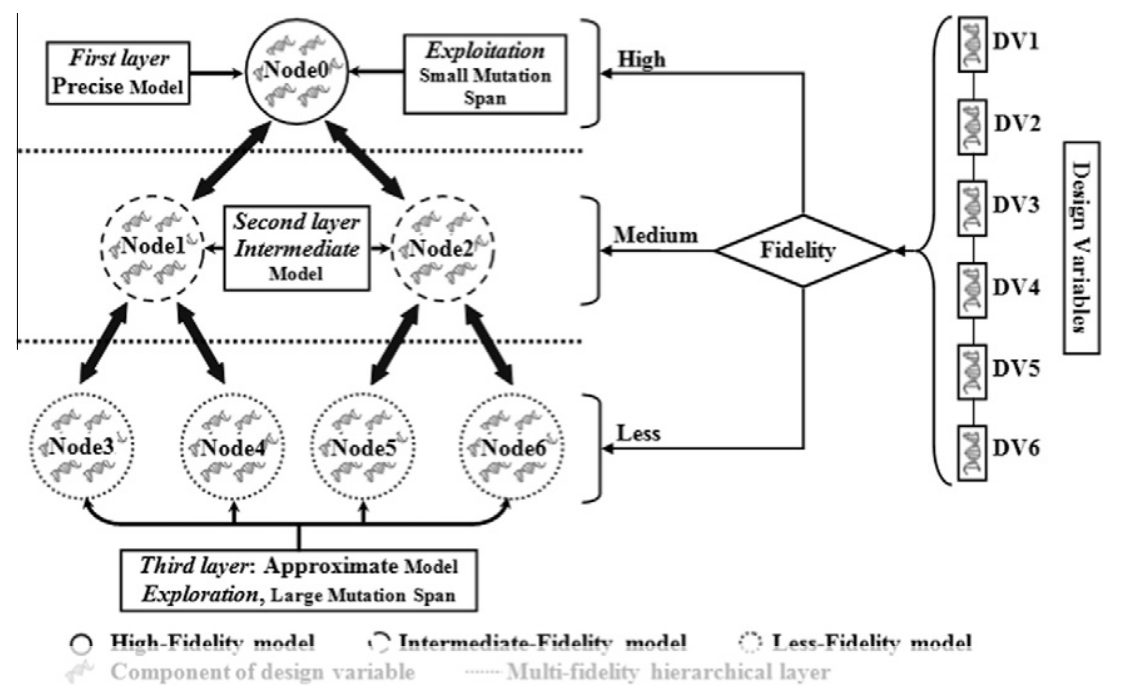

Fig. 1. Topology of HAPMOEA.

concepts of Nash-equilibrium and Pareto optimality are implemented for Hybrid-Game.

The evolutionary algorithm used in this paper is based on

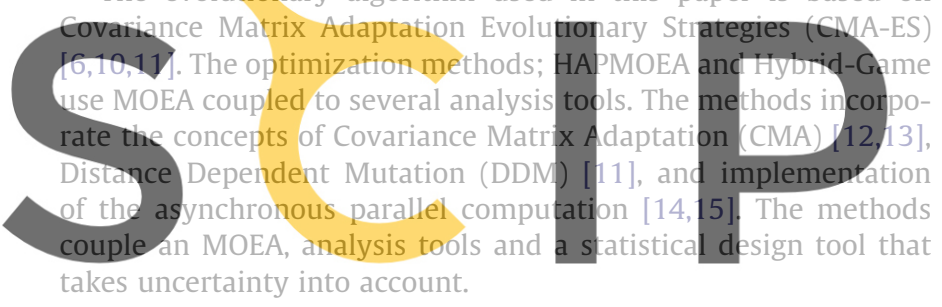

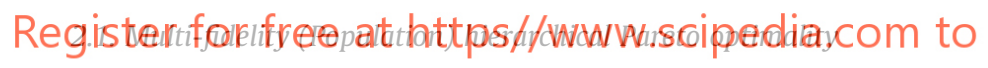

The first method multi-fidelity hierarchical Pareto optimality [16] uses three layers of hierarchical Pareto optimal game and is shown in Fig. 1. The optimiser has capabilities to handle multifidelity/physics models for the solution. There are three layers and seven different populations in HAPMOEA; the first layer (Node0: fine-grid population) concentrates on the refinement of solutions. The third layer (Node3-Node6: four coarse-grid populations) uses approximate model therefore these populations are entirely devoted to exploration. The second layer (Node1 and Node2: two intermediate-grid populations) is to compromise solutions between exploration (third layer) and exploitation (first layer). There is also a migration operation after predefined number of function evaluations; individuals migrate up and down from third to first layer and from first to third layer during the optimization. As an example, if the problem considers six design variables (DV1DV6); each Pareto-game population at each layer evaluates the same and all fitness/objective function, and considers the whole design variable span (DV1-DV6). The topology of HAPMOEA is normally fixed in the authors' test cases for multi-objective and multidisciplinary designs. Details of HAPMOEA can be found in Ref. [6].

\subsection{Hybrid-Game (Hybrid-Nash)}

The Hybrid-Game uses the concepts of Nash-Game and Pareto optimality and hence it can simultaneously produce Nash-equilibrium and a set of Pareto non-dominated solutions [9]. The reason for hybridisation of the concepts of Nash-Game and Pareto optimality is to cover each other's major drawbacks; Nash-Game has a fast search but only one global solution (Nash-equilibrium) while standard EAs based on Pareto optimality generally require a large number of function evaluations to find optimal non-dominated solutions. Nash-Game hereinspeeds up to search one of the global
solutions which will be seeded to the Pareto-Game population at
every generation or after a predefined sequential function evalua-
tion. Each Nash-Player has its own design criteria and uses its own
optimization strategy. Therefoye the Hybrid-Game can accelerate
NiOEA to find Pareto optimal solutions based on the elite design
obtained by the Nash-Game.

Fig. 2a shows the hybridisation between HAPMOEA and NashGame, and one example of the Hybrid-Game topology. The Hy-

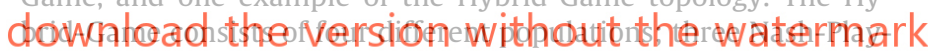
ers (NP1, NP2 and NP3) and one Pareto-Player/Global-Player (Node0: high fidelity/resolution - precise model from HAPMOEA). The Hybrid-Game iocates the Pareto-Player/Giobal-Player at the core of Nash-Game and the elite designs obtained by Nash-Players will be seeded to the Pareto-Player/Global-Player at every generation or after a predefined number of function evaluations. This optimization mechanism allows the Pareto-Player/Global-Player to accelerate to find Pareto non-dominated solutions or global solutions. The topology of Hybrid-Game represents a top view of a trigonal pyramid instead of a two dimensional hierarchical pyramid shape. The topology of Hybrid-Game is flexible; if there are four Nash-Players then the topology will be a quadrangular pyramid.

Fig. 2b shows an example of design variables (DV1-DV6) distribution in a Hybrid-Game. The distribution of design variables is as follows; Nash-Player1 (black circle) only considers black square design components (DV1, DV4), DV2 and DV5 are considered by Nash-Player2 (hidden line circle). Nash-Player3 (dot line circle) considers DV3 and DV6. The Pareto-Player (center line circle) considers the complete design variable span (DV1-DV6). The elite designs (DV1-DV6) obtained by Nash-Players (N-Player1, N-Player2 and N-Player3) will be seeded to the population of Pareto-Player (P-Player) after each generation or after a predefined number of function evaluations has been reached during the optimization. The Hybrid-Game decomposes one design problem into three simpler design problems for Nash-Game which consists of three Nash-Players (N-Player1, N-Player2 and N-Player3). The ParetoPlayer (P-Player) will use elite designs (DV1-DV6) obtained by Nash-Game to find Pareto non-dominated solutions. Therefore, 

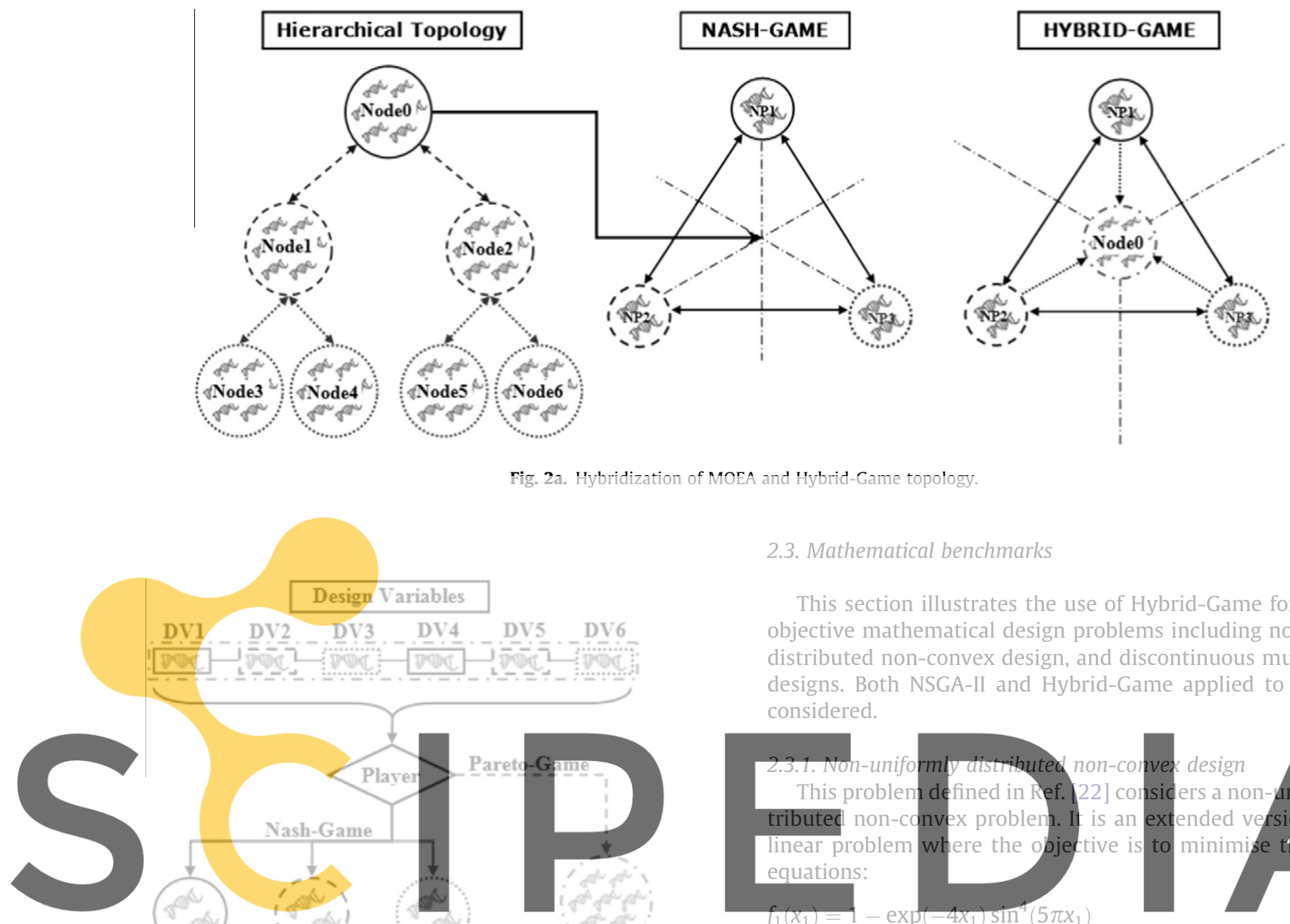

2.3. Mathematical benchmarks

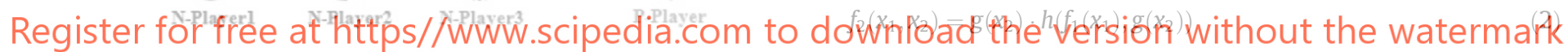 \\ where $0 \leqslant x_{1}, x_{2} \leqslant 1$}

This section illustrates the use of Hybrid-Game for two multiobjective mathematical design problems including non-uniformly distributed non-convex design, and discontinuous multi-objective designs. Both NSGA-II and Hybrid-Game applied to NSGA-II are considered.

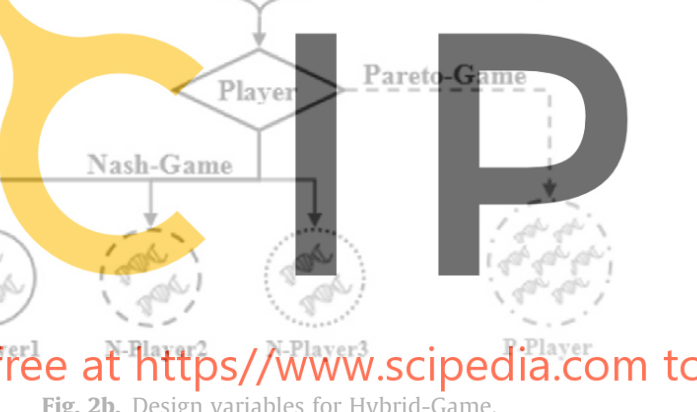

Pareto-Player can accelerate its searching diversity to true Pareto front or global solutions. The Hybrid-Game can be applied to non-generational EAs such as HAPMOEA as well as to generation based approaches.

Lee et al. [17] studied the concept of Hybrid-Game (Pareto and Nash) coupled to a well-known MOEA; Non-dominating Sort Genetic Algorithm II (NSGA-II) [18]. Their research shows that the Hybrid-Game improves the NSGA-II performance by $80 \%$ when compared to the original NSGA-II. In addition, Lee et al. [19] hybridised NSGA-II with Nash-Game strategy to study a role of NashPlayers in Hybrid-Game by solving multi-objective mathematical test cases; non-uniformly distributed non-convex, discontinuous and mechanical design problem. Their research also shows that HAPMOEA can be also hybridised with Nash-Game to solve a real-world robust multidisciplinary design problem. Numerical results show that the Hybrid-Game improves 70\% of HAPMOEA performance while producing better Pareto optimal solutions.

It should be noted that the Nash-equilibrium solution can be one of Pareto front members since the elite designs obtained by Nash-Players are seeded to the Pareto-Player population however the Nash-equilibrium will not be part of the Pareto-front if the Nash-equilibrium is dominated by Pareto-Player solutions. The validation of Hybrid-Game and HAPMOEA can be found in Refs. $[9,17,19-21]$. $g\left(x_{2}\right)=\left\{\begin{array}{ll}4-3 \exp \left(-\left(\frac{x_{2}-0.2}{0.02}\right)^{2}\right) & \text { if } 0 \leqslant x_{2} \leqslant 0.4 \\ 4-3 \exp \left(-\left(\frac{x_{2}-0.7}{0.2}\right)^{2}\right) & \text { if } 0.4 \leqslant x_{2} \leqslant 1\end{array} h\left(f_{1}, g\right)=\left\{\begin{array}{ll}1-\left(\frac{j_{1}}{g}\right) & \text { if } f_{1} \leqslant g \\ 0 & \text { otherwise }\end{array} \quad \alpha=4\right.\right.$

Random solutions are shown in Fig. 3a. Fig. 3b compares the convergence obtained by NSGA-II and Hybrid-Game coupled to NSGA-II (Hybridised NSGA-II). The optimization is stopped after 50 generations with a population size of 100 . It can be seen that the NSGA-II requires more function evaluations (marked with red circle) while the Hybridised NSGA-II has already capture the true Pareto front.

The initial populations obtained by NSGA-II and Hybridised NSGA-II are shown in Figs. $4 a$ and $4 b$ respectively where the NSGA-II has a better non-dominated solutions (nine members) distribution than the Hybridised NSGA-II with seven non-dominated solutions. However, it can be seen that the Nash-Players of Hybridised NSGA-II found the elite solution (mark with circle) which dominates Pareto members 1-5 of Pareto-Game. This Nash elite design will be seeded to the population of Pareto-Game and will become a Pareto member 1 then the Pareto-Game will generate the candidates based on the Nash elite design. This is the reason why the Hybridised NSGA-II had faster convergence than NSGA-II.

\subsubsection{Discontinuous $\mathrm{MO}$ (TNK) design}

The problem TNK proposed in Ref. [23] considers minimisation of the following equation:

$f_{1}\left(x_{1}\right)=x_{1}$ and $f_{2}\left(x_{2}\right)=x_{2}$ 


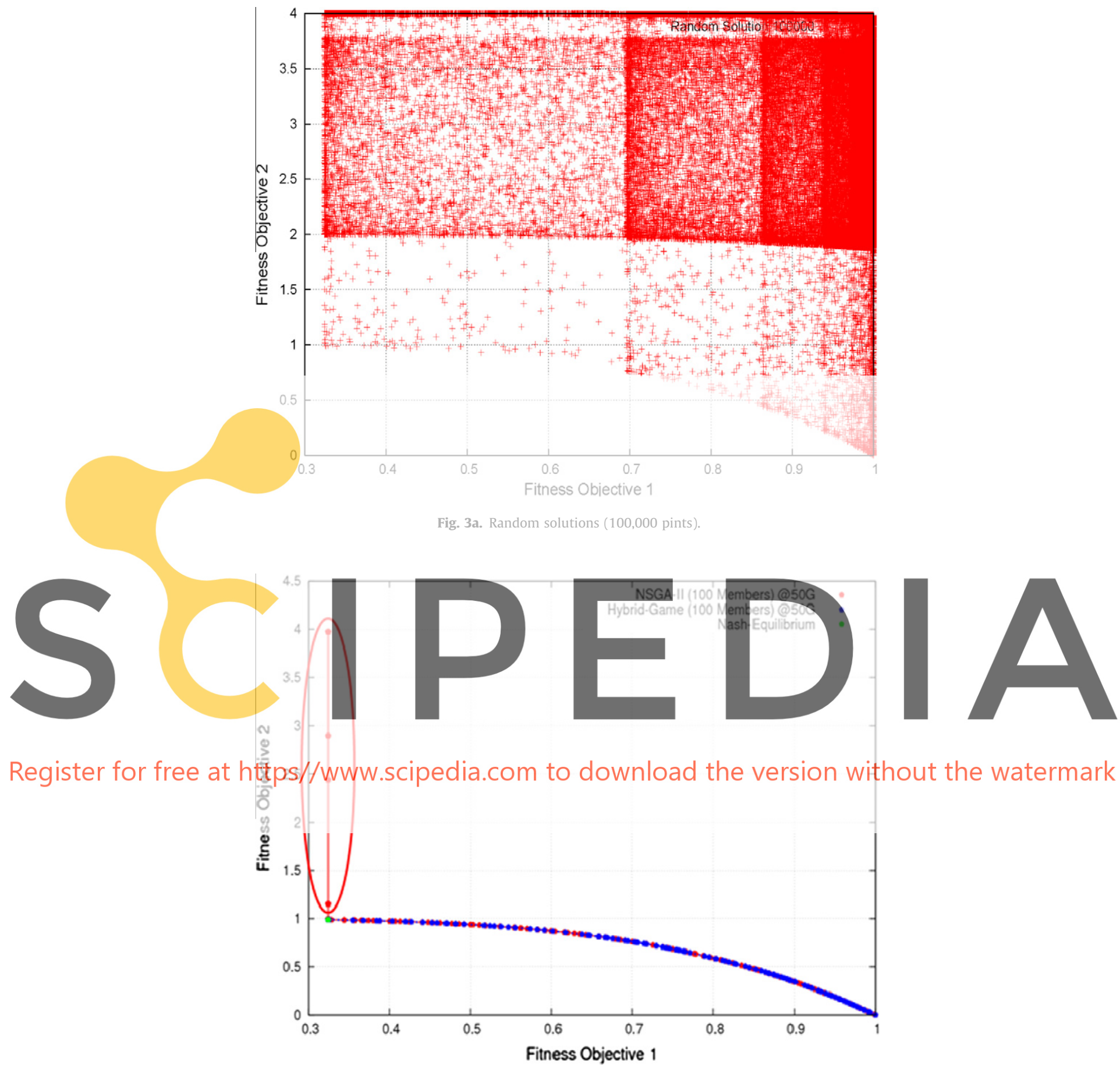

Fig. 3b. Comparison of Pareto fronts.

Subject to

$C_{1}\left(x_{1}, x_{2}\right)=-x_{1}^{2}-x_{2}^{2}+1+0.1 \cos \left(16 \arctan \frac{x_{1}}{x_{2}}\right) \leqslant 0$

$C_{2}\left(x_{1}, x_{2}\right)=\left(x_{1}-0.5\right)^{2}+\left(x_{2}-0.5\right)^{2} \leqslant 0.5$

where $0 \leqslant x_{1}, x_{2} \leqslant \pi$

Random solutions are shown in Fig. 5a. Fig. 5b compares the convergence obtained by NSGA-II and the Hybridised NSGA-II. The optimization is stopped after 100 generations with a popula- tion size of 100. It can be seen that the NSGA-II need more function evaluations to find Pareto members in the Section-A while the Hybrid-Game converged to the true Pareto front.

\subsection{Algorithms for HAPMOEA and Hybrid-Game}

Algorithms for HAPMOEA and Hybrid-Game are shown in Figs. $6 a$ and $6 b$ to further illustrate the HAPMOEA and the Hybrid-Game concepts. It is assumed that the problem considers the fitness function $f=\min \left(x_{1}, x_{2}, x_{3}\right)$ as an example. 


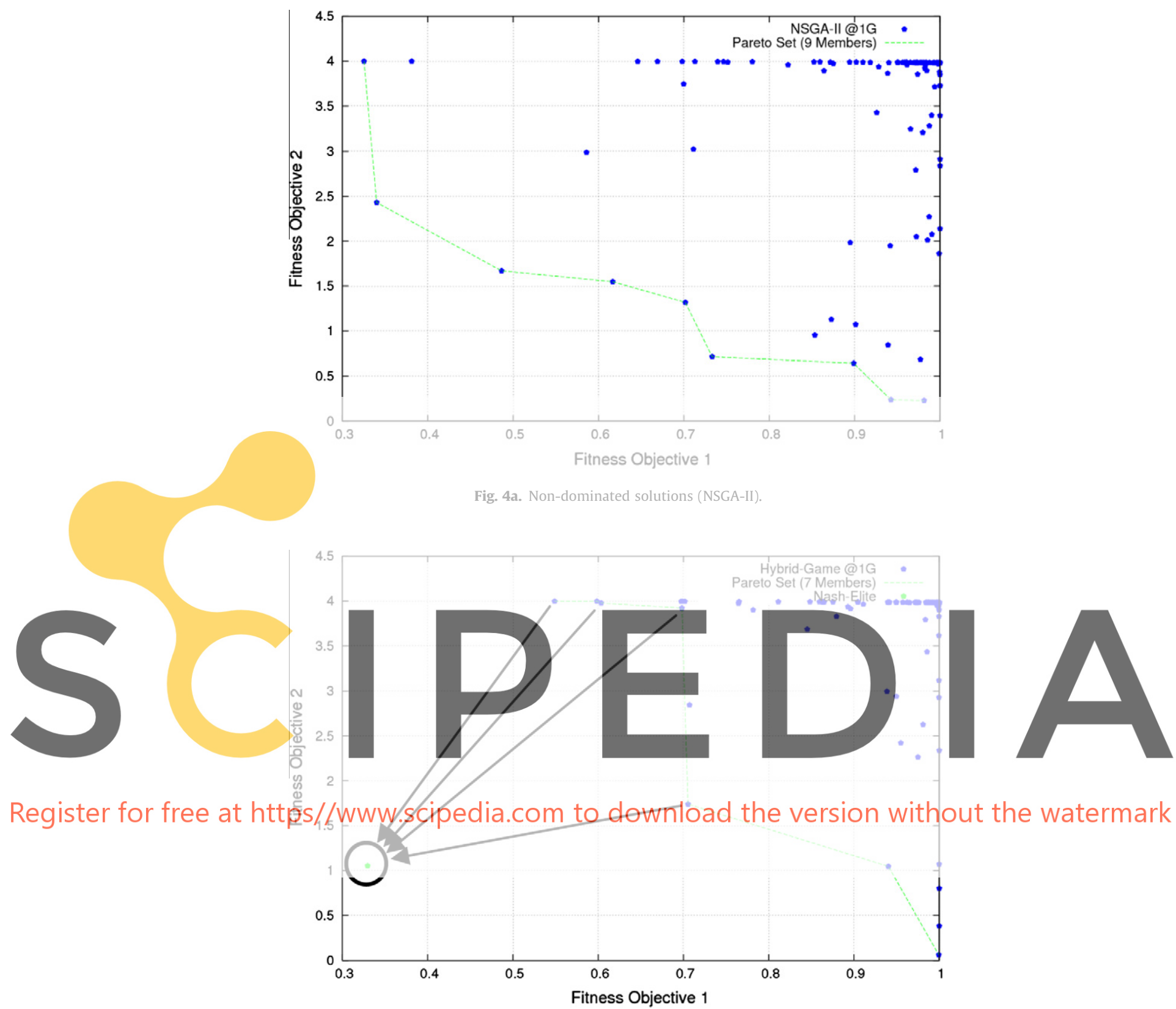

Fig. 4b. Non-dominated solutions (Hybridised NSGA-II).

\subsubsection{HAPMOEA-L3 (Fig. 6a)}

The method has eight main steps as follows;

- Step 1: Define population size and number of generation for hierarchical topology (Node0-Node6), number of design variables $\left(x_{1}, x_{2}, x_{3}\right)$ and their design bounds, model quality for each layer (Layer1 (Node0): precise, Layer2 (Node1, Node2): intermediate, Layer3 (Node3-Node6): least precise).

- Step 2: Initialize seven random populations for Node0-Node6.

while termination condition (generation or elapsed time or predefined fitness value).

- Step 3: Generate offspring using mutation or recombination operations.

- Step 4: Evaluate each offspring and compute fitness functions.
- Step 4.1: Evaluate offspring for each node in terms of precise, compromise, least precise.

- Step 5: Sort each population for each node based on its fitness.

- Step 6: Replace best individual into non-dominated population of each node.

end

- Step 7: Designate results for the optimization; Pareto optimal front obtained by Node0 at first layer (precise model) for multi-objective design problem otherwise plot convergence of optimization based on best-so-far individual.

- Step 8: Do post-optimization process; if problem considers aerodynamic wing design Mach sweep will be plotted corresponding to objective (CD, CL, L/D). 


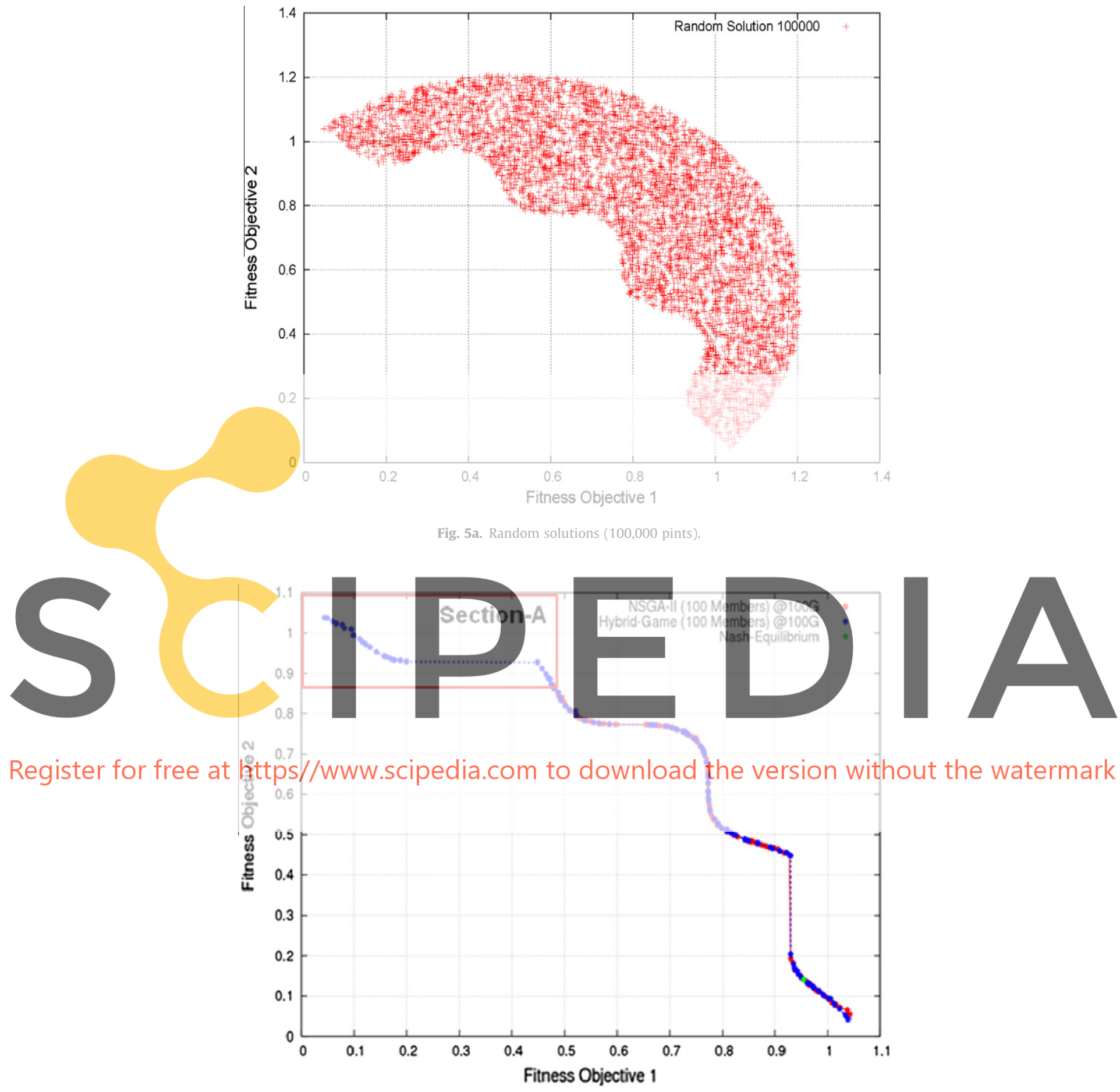

Fig. 5b. Comparison of Pareto fronts for TNK.

\subsubsection{Hybrid-Game (Fig. 6b)}

The method has eight main steps as follows;

- Step 1: Define population size, number of generation or function evaluations for each Nash-Players (N-Player1, N-Player2, N-Player3) and Pareto Player (P-Player), dimension of decision variables $\left(x_{1}, x_{2}, x_{3}\right)$ and their design bounds. Split decision variables for each player (N-Player1: $x_{1}$, N-Player2: $x_{2}$, N-Player3: $x_{3}$, P-Player: $\left.x_{1}, x_{2}, x_{3}\right)$.

- Step 2: Initialize random population for each player.
- while termination condition (generation or elapsed time or predefined fitness value).

- Step 3: Generate offspring using mutation or recombination operations.

- Step 4: Evaluate each offspring and compute its fitness functions.

- Step 4.1: Evaluate offspring in Nash-Game.

- N-Player1: Use $x_{1}$ with design variables $x_{2}, x_{3}$ fixed by $\mathrm{N}$ Player2 and N-Player3. 


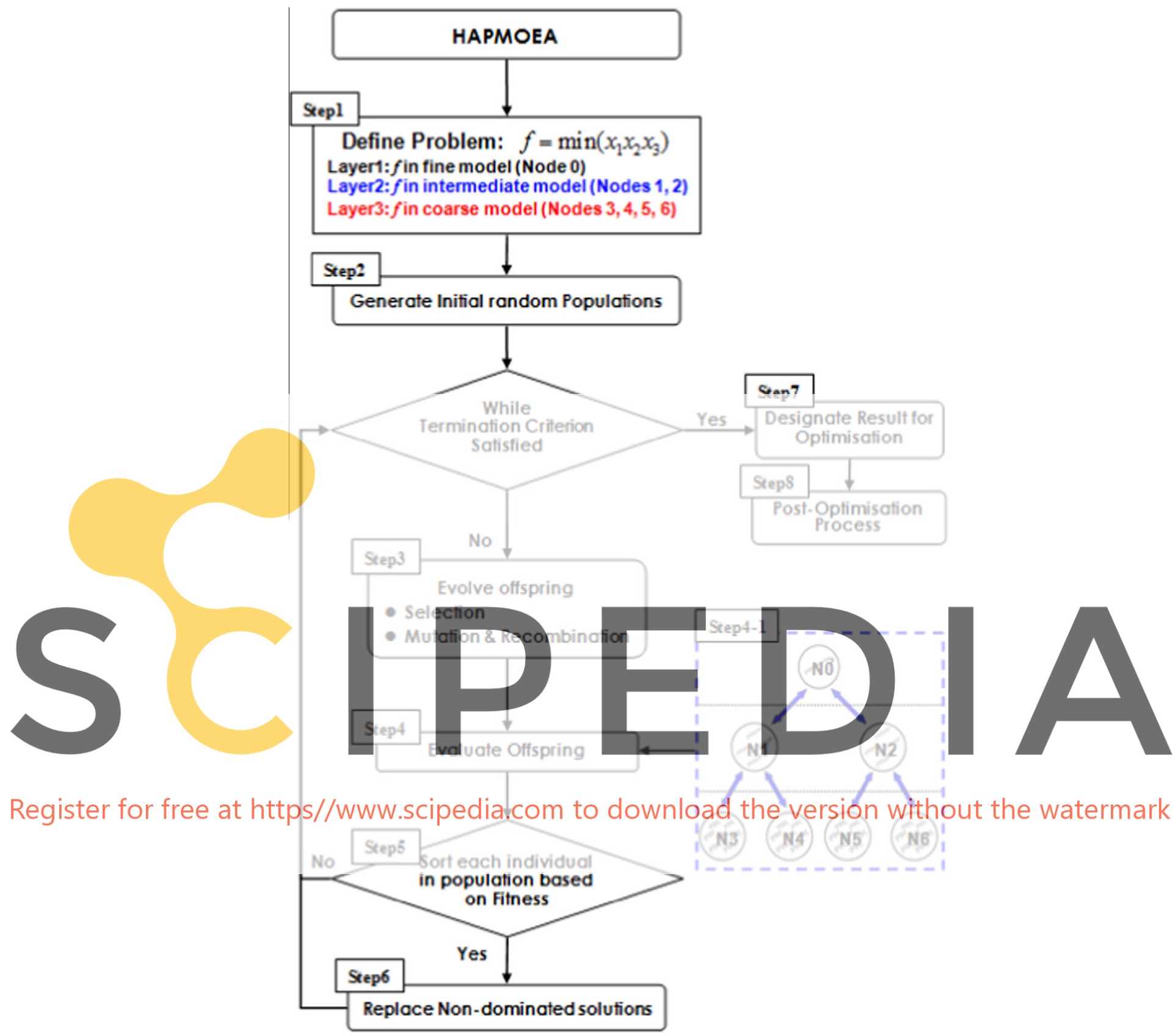

Fig. 6a. Algorithm of HAPMOEA.

- N-Player2: Use $x_{2}$ with design variables $x_{1}, x_{3}$ fixed by $\mathrm{N}$ Player1 and N-Player3.

- N-Player3: Use $x_{3}$ with design variables $x_{1}, x_{2}$ fixed by $\mathrm{N}$ Player1 and N-Player2.

- Step 4.2: Evaluate offspring for P-Player.

- if (the first offspring at each generation is considered)

- P-Player: use elite design $\left(x_{1}^{*}, x_{2}^{*}, x_{3}^{*}\right)$ obtained by Nash-Game at Step 4.1.

- else

- P-Player: use $\mathrm{x}_{1}, \mathrm{x}_{2}, \mathrm{x}_{3}$ obtained by mutation or recombination operation as default.

- Step 5: Sort each population for each player based on its fitness.

- Step 6: Replace the non-dominated individual into best population for P-Player.

- end

- Step 7: Designate results.
P-Player: Plot Pareto optimal front for multi-objective design problem otherwise plot convergence of optimization based on best-so-far individual.

Nash-Game: plot Nash-equilibrium obtained by N-Player1, NPlayer2, N-Player3.

- Step 8: Do post-optimization process; if problem considers aerodynamic wing design Mach sweep will be plotted corresponding to objective (CD, CL, L/D).

\section{Aerodynamic analysis tools}

The first aerodynamic analysis tool used in this paper is a potential flow solver. The solver is used for analysing inviscid, isentropic, transonic shocked flow past 3D swept wing configurations [24]. 


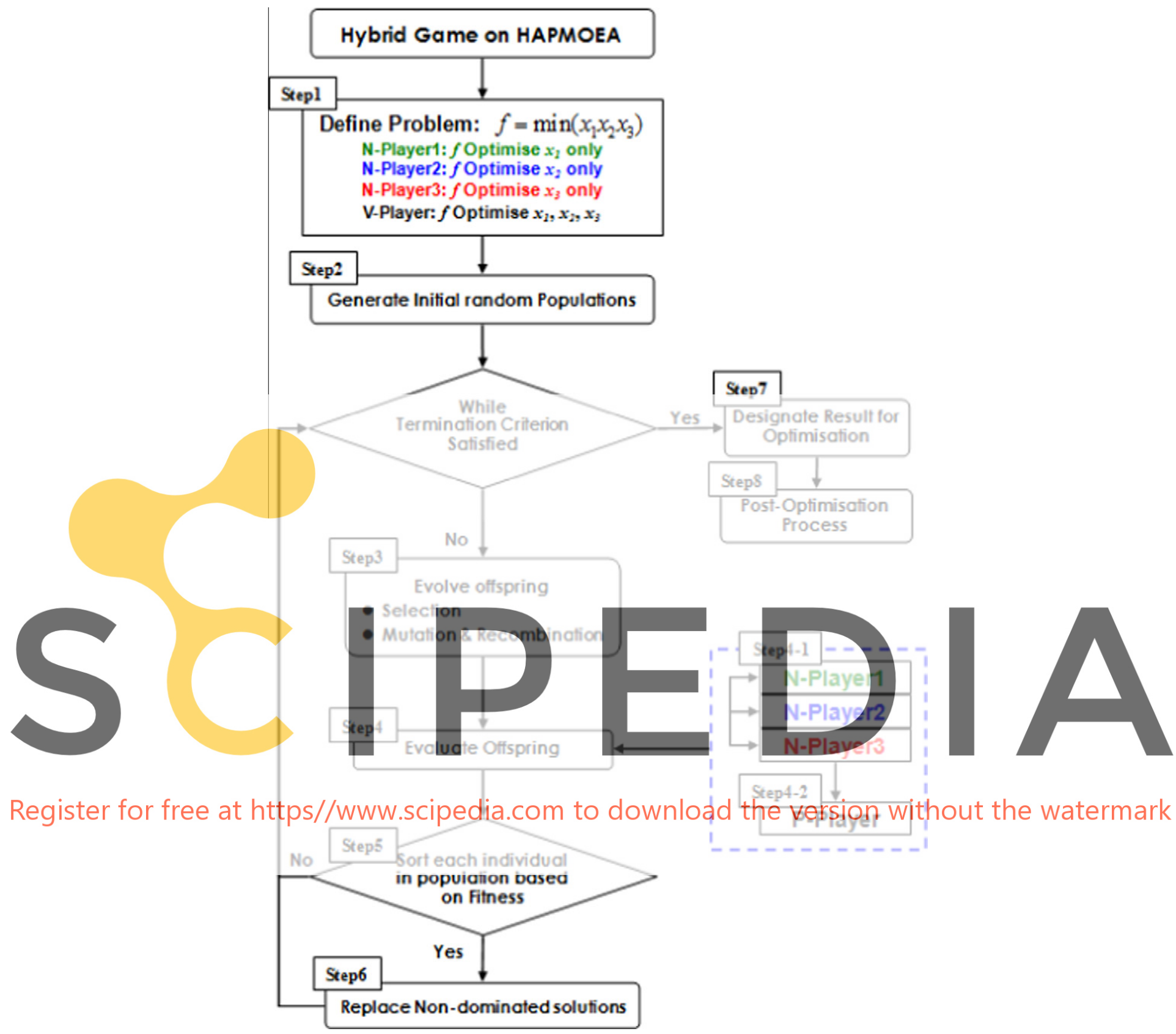

Fig. 6b. Algorithm of Hybrid-Game.

The second analysis tool is for friction drag which is externally computed by utilizing the program FRICTION code [25]. The code provides an estimate of the laminar and turbulent the skin friction suitable for use in aircraft preliminary design. Details on the validation of the potential flow solver can be found in Ref. [21] where it is shown that the results obtained by the potential flow solver are in good agreement with experimental data.

\section{Real world design problem: MO design optimization of UCAS}

In this section, the Hybrid-Game is used to show the benefit of using Nash-game and Pareto-game simultaneously. To do so, the results obtained by Hybrid-Game will be compared to the results obtained by HAPMOEA. It is shown that the Hybrid-Game has the potential to produce high quality solutions and to reduce computational expense.

\subsection{Formulation of design problem}

Unmanned (Combat) Aerial Systems (UCASs) have broad applicability for a number of civilian and military missions [26,27]. The type of vehicle considered in this section is a Joint Unmanned Combat Air Vehicle (J-UCAV) that is similar in shape to the Northrop Grumman X-47B [28]. This test case considers the design optimization of the UCAV wing aerofoil sections and planform geometry. The objectives are to maximize both mean values of lift coefficient $\left(\overline{C_{L}}\right)$ and lift to drag ratio $(\overline{L / D})$ to maximize a manoeuvrability and range of UCAV. The baseline UCAV is shown in Figs. 7a and 7b.

The wing planform shape is assumed as an arrow shape with jagged trailing edge. The aircraft maximum gross weight is approximately $46,396 \mathrm{lb}(21,045 \mathrm{~kg})$ and the empty weight is $37,379 \mathrm{lb}$ $(16,955 \mathrm{~kg})$. The design parameters for the baseline wing configuration are illustrated in Fig. $7 \mathrm{~b}$ and Table 1 . In this test case, the fuselage is assumed from $0 \%$ to $25 \%$ of the half span. The crank 


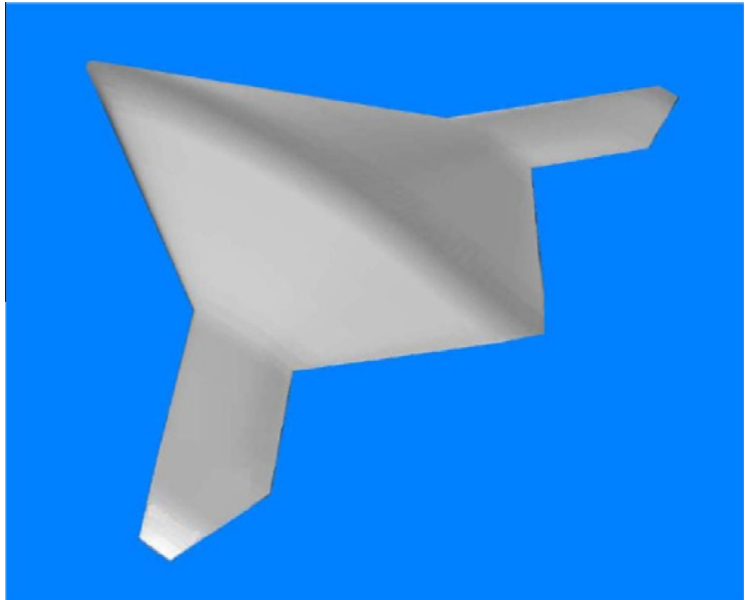

Fig. 7a. Baseline design (3D-view).

positions are at $46.4 \%$ and $75.5 \%$ of the half span. The inboard and outboard sweep angles are $55^{\circ}$ and $29^{\circ}$. Inboard and outboard taper ratios are $20 \%$ and $2 \%$ of the root chord respectively.

It is assumed that the baseline wing design contains three types of airfoils; the NACA 66-021 and NACA 67-1015 are located at inboard (root and crank1) and the NACA 67-008 are located at the outboard sections (crank2 and tip). These airfoils are shown in Fig. 8. The maximum thickness at root section is $21 \%$ of the root

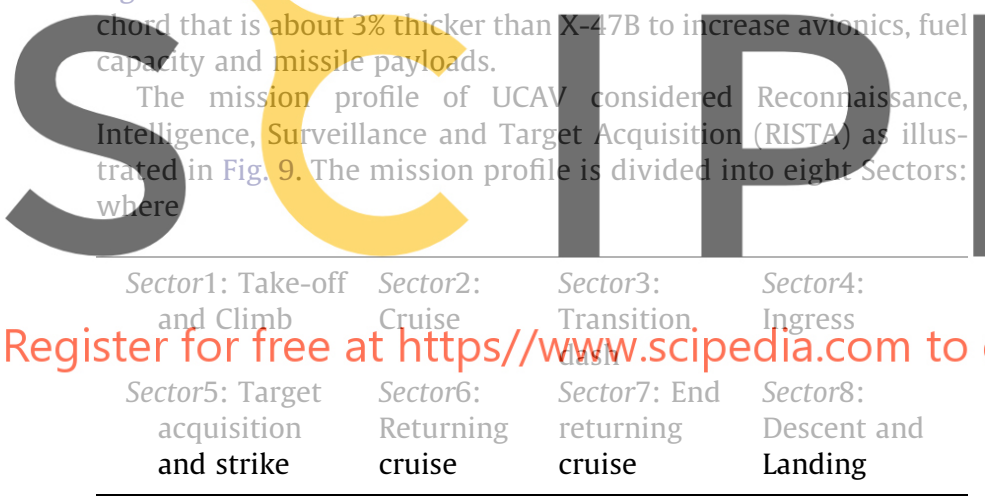

Table 1

Baseline UCAV wing configurations.

\begin{tabular}{lllllllll}
\hline$A R$ & $b$ & $\Lambda_{R-C 1}$ & $\Lambda_{C 1-C 2}$ & $\Lambda_{C 2-T}$ & $\lambda_{C 1}$ & $\lambda_{C 2}$ & $\lambda_{T}$ & $\Gamma_{\text {Overall }}$ \\
\hline 4.377 & $18.9 \mathrm{~m}$ & $55^{\circ}$ & $29^{\circ}$ & $29^{\circ}$ & 20 & 20 & 2 & $0^{\circ}$ \\
\hline
\end{tabular}

Note: Taper ratio $(\lambda)$ is $\% C_{R o o t}$.

Fig. 10 shows the weight distribution along the mission profile (Sector1-Sector8). The weight between Sector4 and Sector5 is significantly reduced after $80 \%$ of ammunition weight is used for target strike.

In this paper, flight conditions for Sector2-Sector4 are considered for optimization. The minimum lift coefficients $\left(C_{L_{\text {Minimum }}}\right)$ are 0.296 and 0.04 for Sector 2 and Sector 4 respectively as shown in Fig. 11. The baseline design produces $30 \%$ higher lift coefficient at Sector 2 when compared to $C_{L_{\text {Minimum }}}$ while only $7 \%$ higher at Sector4. The aim of this optimization is the improvement of aerodynamic performance $(L / D)$ at Sector 4 while maintaining aerodynamic performance at Sector2.

\subsection{Representation of design variables}

The problem considers design variables for wing and aerofoil design parameters. The aerofoil geometry is represented using Bézier curves with a combination of a mean line and thickness distribution control points. The upper and lower bounds for mean and thickness control points at root, crank1, crank2 and tip sections are

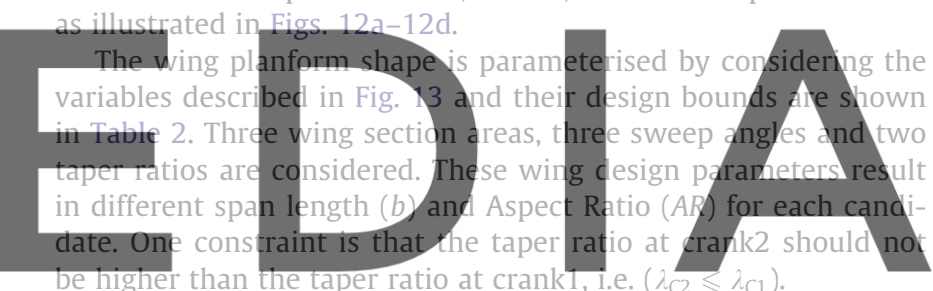
be higher than the taper ratio at crank1, i.e. $\left(\lambda_{c 2} \leqslant \lambda_{c 1}\right)$.

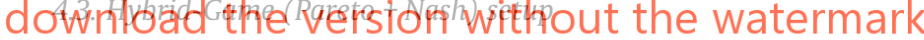

In this paper, Hybrid-Game employs five Nash-Players and one Pareto-Player as shown in Table 3. The Pareto-Player of HybridGame uses all 76 design variables for the aerofoil sections and wing planform. Aerofoil sections at root, crank1, crank2 and tip are opti-

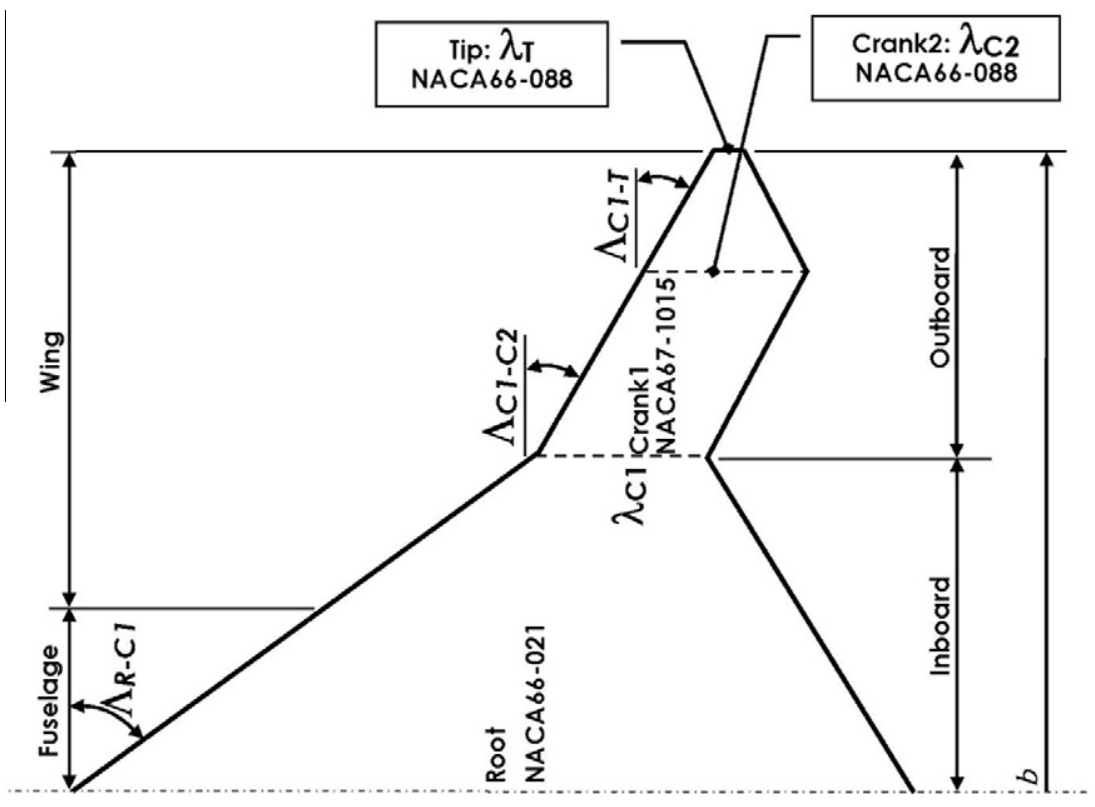

Fig. 7b. Baseline UCAV configuration. 


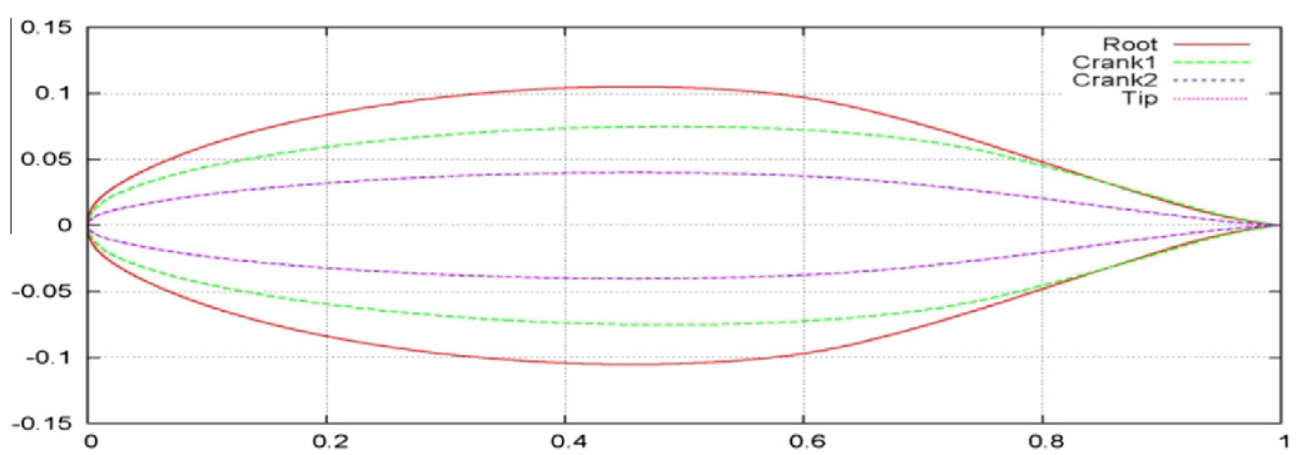

Fig. 8. Baseline UCAV wing airfoil sections.

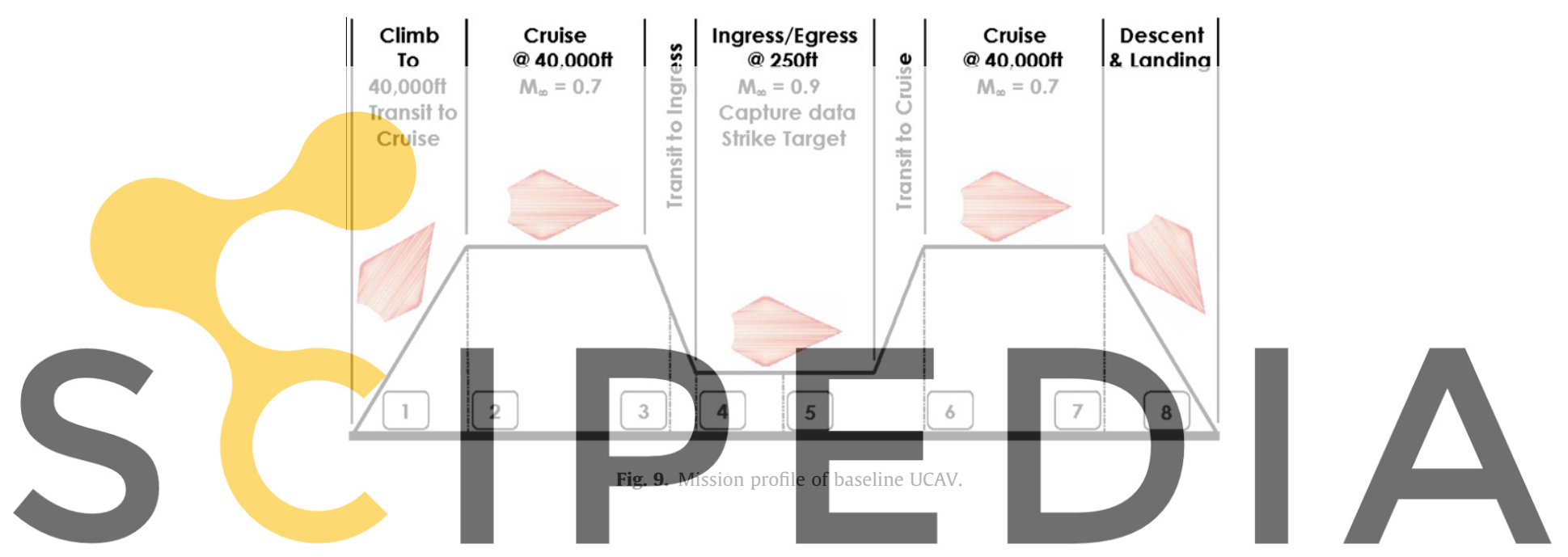

Register for free at https//www.scipedia.com to download the version without the watermark

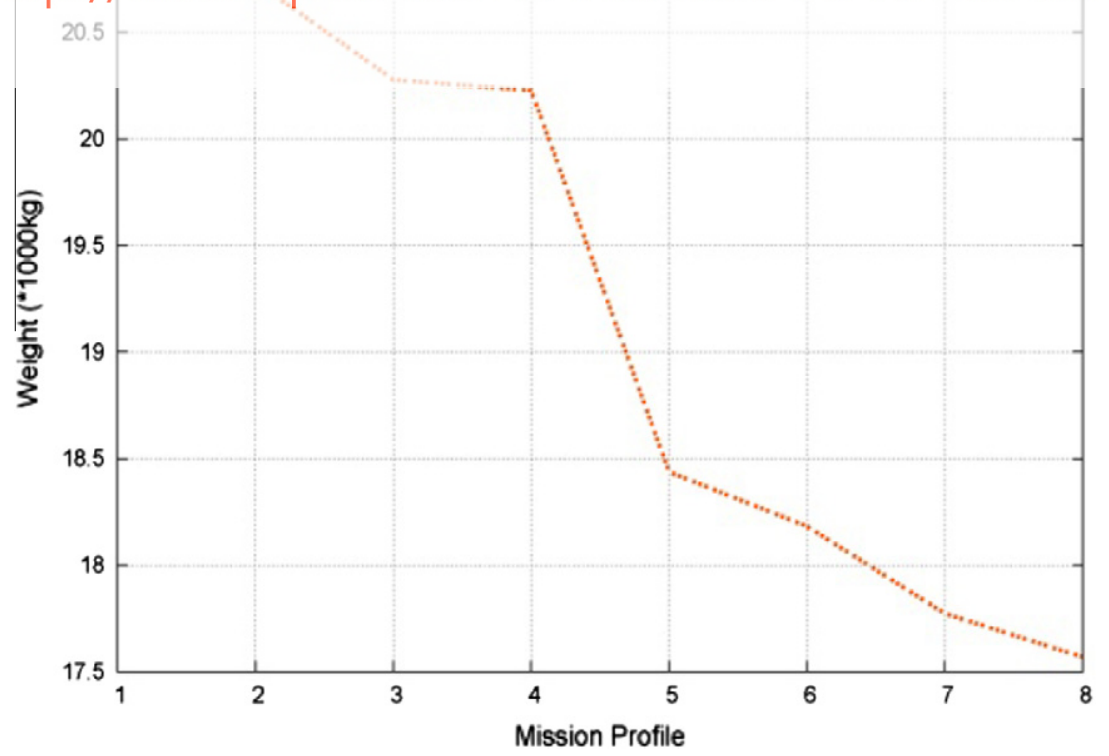

Fig. 10. Weight distribution along the mission.

mized by Nash-Players $1-4$ ( $4 \times 17$ design variables) while NashPlayer5 optimizes wing planform only (eight design variables). In contrast, each node (Node0-6) of HAPMOEA considers all 76 design variables for the aerofoil sections and wing planform.

\subsection{Fitness functions}

Table 4 shows the fitness functions considered by the ParetoPlayer and Nash-Players. The Pareto-Player considers two fitness 


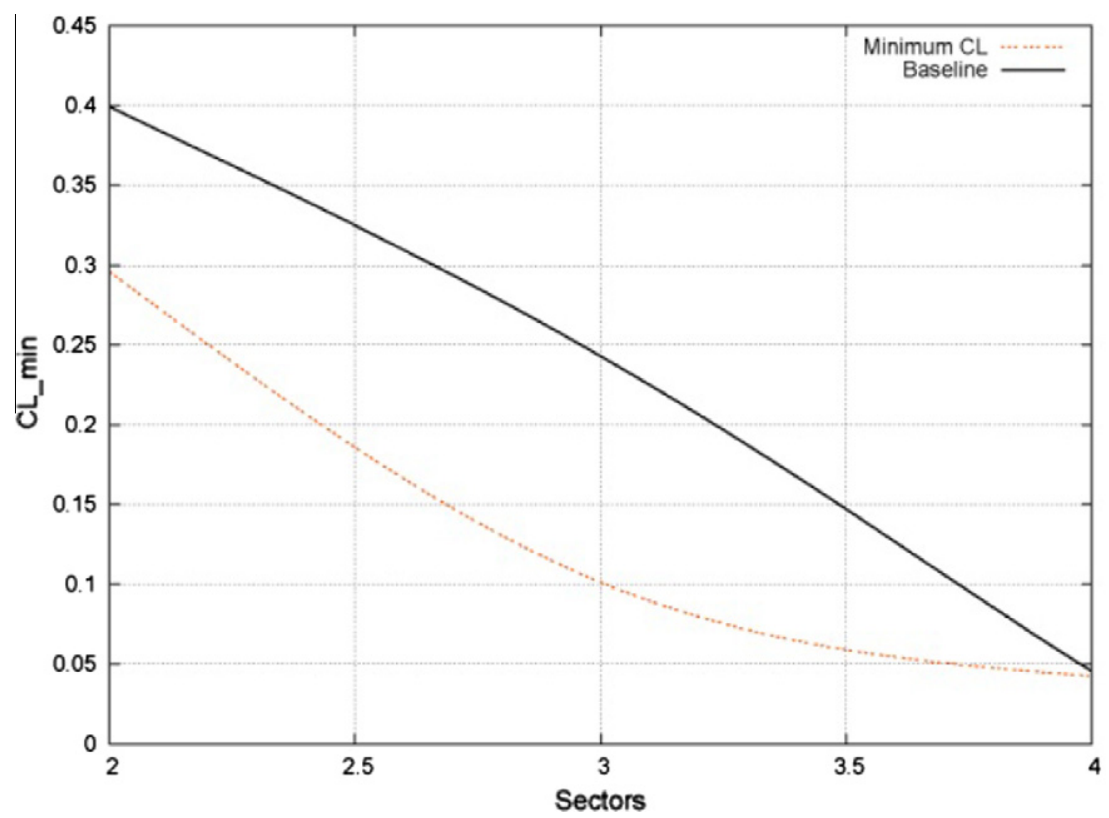

Fig. 11. $C_{L_{\text {Min }}}$ for Sector2-Sector4.

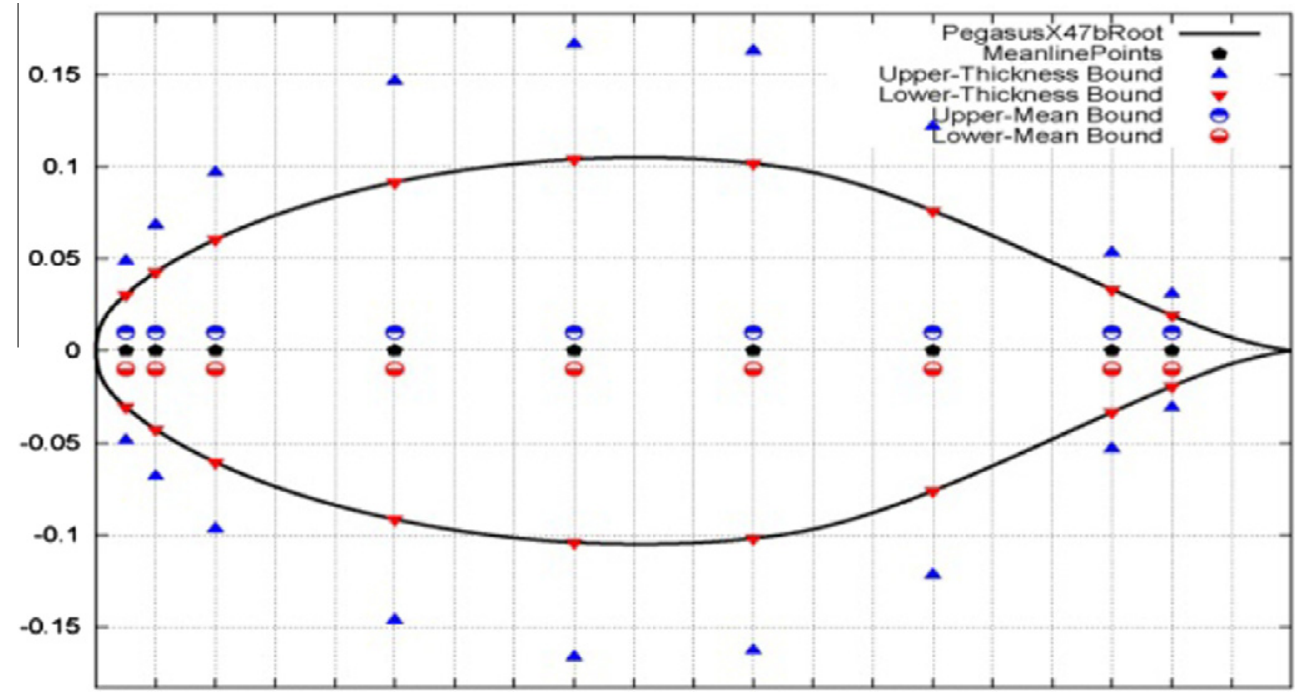

Fig. 12a. Control points at root section.

functions to maximize mean $C_{L}$ and mean $L / D$. The five Nash-Players consider one fitness function to optimize aerofoil sections and wing planform shape. In practice, the Nash-Players look for their elite/best aerodynamic shape for aerofoil sections and wing planform design parameters and these elite design parameters will be seeded to the Pareto-Player to accelerate its convergence. Each node (Node0-Node6) of HAPMOEA uses two fitness functions of Pareto-Player.

The flight conditions are;

- Sector2: $M_{\infty}=0.7, \alpha=6.05^{\circ}$ and altitude $40,000 \mathrm{ft}$. Sector4: $M_{\infty}=0.9, \alpha=0.5^{\circ}$ and altitude $250 \mathrm{ft}$.

\subsection{Interpretation of numerical results}

Both HAPMOEA and Hybrid-Game were run using two $2.4 \mathrm{GHz}$ processors. The HAPMOEA algorithm was allowed to run approximately for 6667 function evaluations and took 200 h. The Hybrid-
Game (Hybrid MOEA) algorithm was run approximately for 1300 function evaluations and took $50 \mathrm{~h}$. The Pareto fronts obtained by HAPMOEA and Hybrid-Game are compared to the baseline design in Fig. 14. It can be seen that Hybrid-Game produces better nondominated solutions in terms of fitness function 1 and 2 when compared to HAPMOEA.

Table 5 compares the Pareto optimal solutions obtained by HAPMOEA and Hybrid-Game. It can be seen that Hybrid-Game produces twice the value of the lift coefficient $\left(C_{L}\right)$ while slightly better results for inverse mean lift to drag ratio $(L / D)$ when compared to the Pareto members obtained by HAPMOEA.

Table 6 compares the quality of drag coefficient obtained by HAPMOEA and Hybrid-Game using the uncertainty mean and variance statistical formulas. It can be seen that Pareto members of Hybrid-Game produces lower drag at [Sector2:Sector4] while Pareto members from HAPMOEA produce stable drag.

The Sector sweep is plotted with the lift coefficient and lift to drag ratio as shown in Figs. 15a and 15b. The range of Sector sweep 


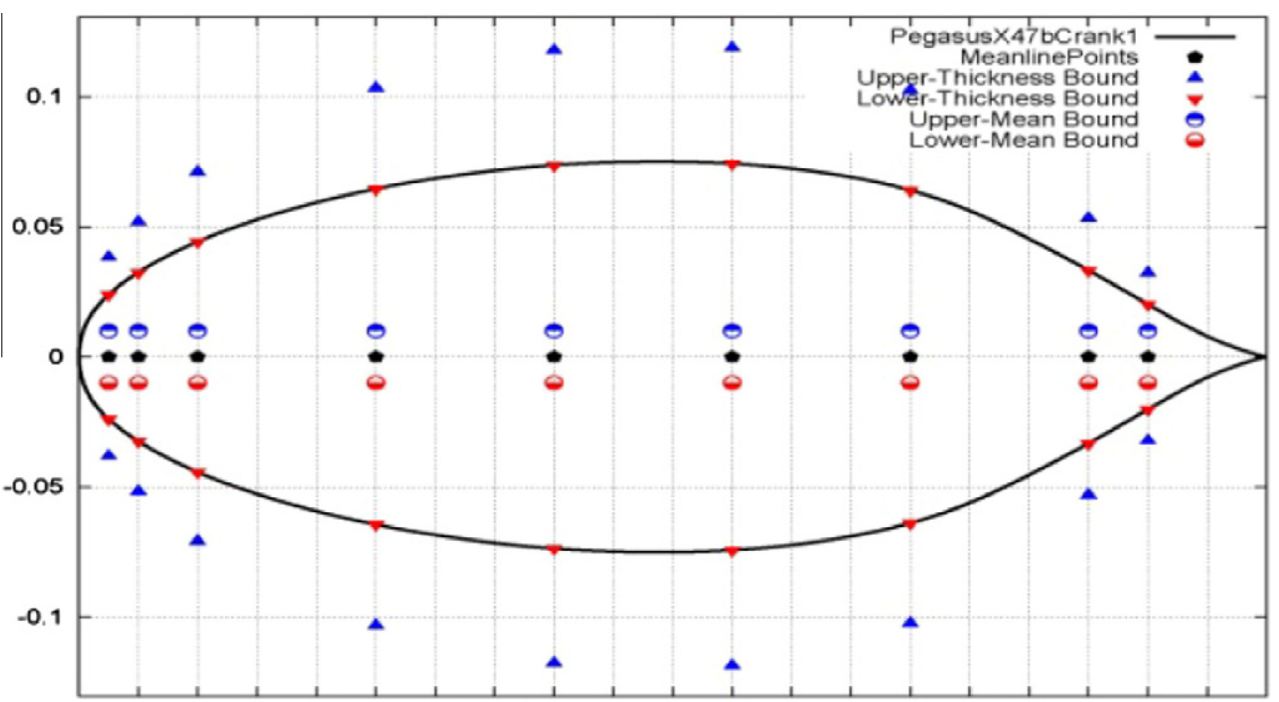

Fig. 12b. Control points at crank1 section.

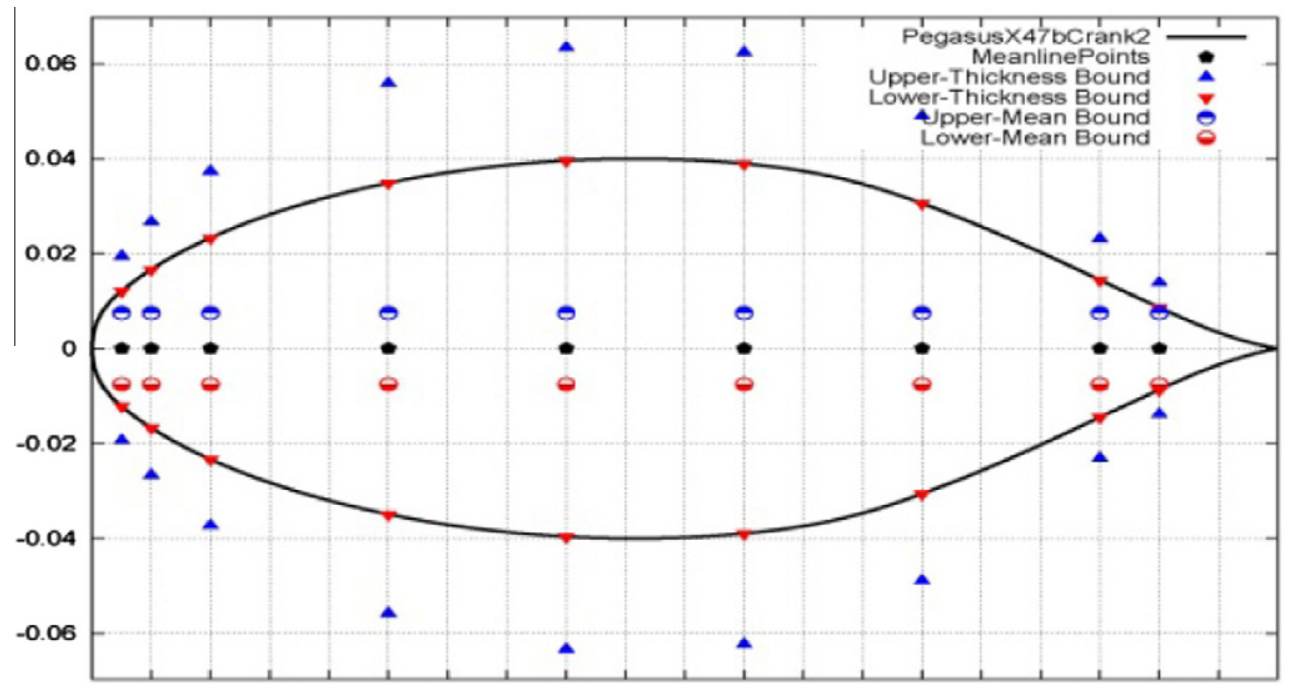

Fig. 12c. Control points at crank2 section.

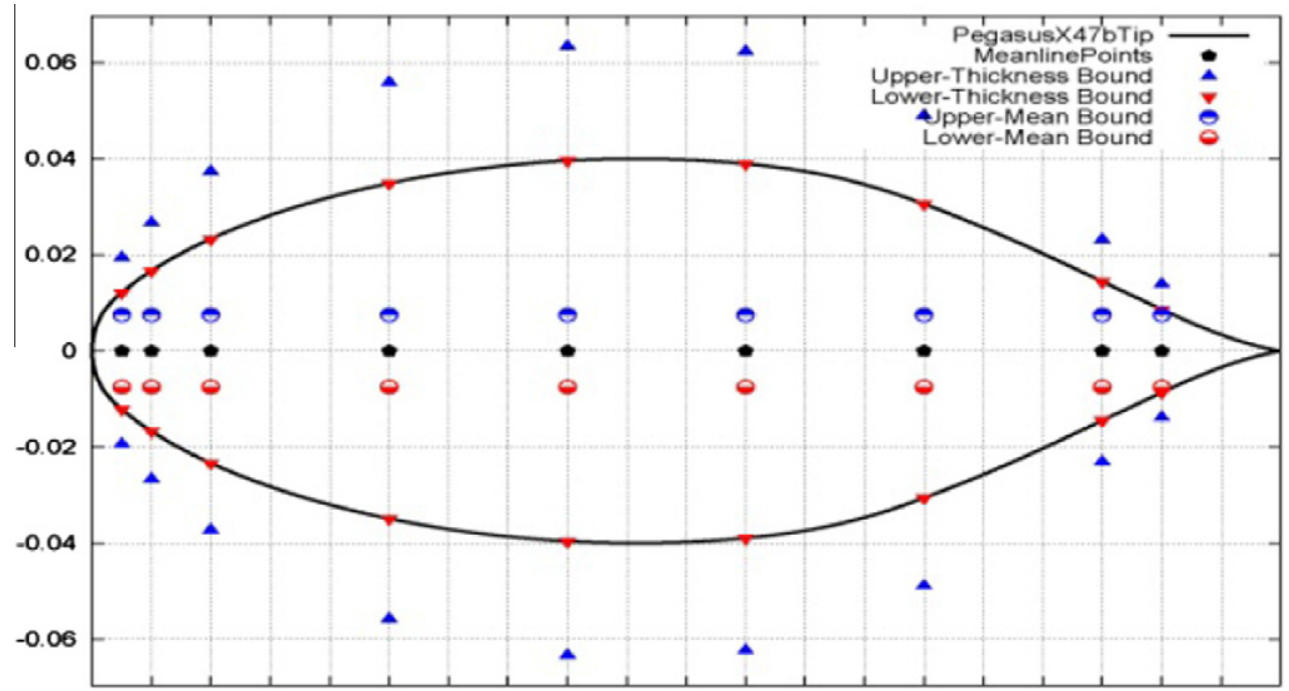

Fig. 12d. Control points at tip section. 


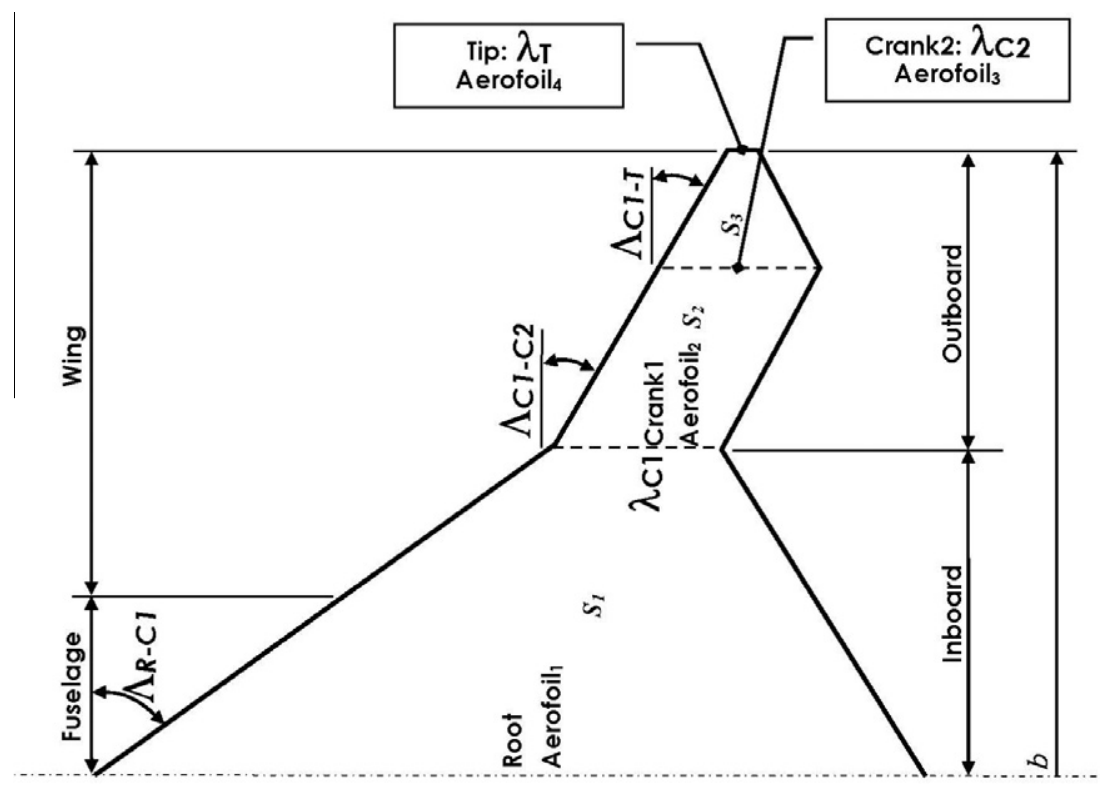

Fig. 13. Wing planform design variables.

Table 2

Wing planform design bounds.

\begin{tabular}{|c|c|c|c|c|c|c|c|c|}
\hline Variables bounds & $S_{1}$ & $S_{2}$ & $S_{3}$ & $\Lambda_{R-C 1}\left(^{\circ}\right)$ & $\Lambda_{\mathrm{C} 1-\mathrm{C} 2}\left(^{\circ}\right)$ & $\Lambda_{C 2-T}\left({ }^{\circ}\right)$ & $\lambda_{C 1}$ & $\lambda_{\mathrm{C} 2}$ \\
\hline Lower & 50.46 & 10.09 & 5.05 & 49.5 & 25 & 25 & 0.15 & 0.15 \\
\hline Upper & 63.92 & 16.82 & 10.09 & 60.5 & 35 & 35 & 0.45 & 0.45 \\
\hline
\end{tabular}

Note: Area $(S)$ is in $\mathrm{m}^{2}$ and one geometrical constraint is applied $\lambda_{\mathrm{C} 2} \leqslant \lambda_{\mathrm{C} 1}$.

Table 3

Distribution of design variables for Hybrid-Game and HAPMOEA.

\begin{tabular}{|c|c|c|c|c|c|c|c|}
\hline \multirow[t]{2}{*}{ Design variables } & \multicolumn{6}{|c|}{ Hybrid-Game on HAPMOEA } & \multirow[t]{2}{*}{ HAPMOEA } \\
\hline & $\mathrm{N}-\mathrm{P} 1$ & $\mathrm{~N}-\mathrm{P} 2$ & $\mathrm{~N}-\mathrm{P} 3$ & $\mathrm{~N}-\mathrm{P} 4$ & $\mathrm{~N}-\mathrm{P} 5$ & P-Player & \\
\hline Aerofoil $_{\text {Root }}(17$ DVs) & $\sqrt{ }$ & & & & & $\sqrt{ }$ & $\sqrt{ }$ \\
\hline Aerofoil $_{\text {Crank1 }}(17$ DVs) & & $\sqrt{ }$ & & & & $\sqrt{ }$ & $\sqrt{ }$ \\
\hline Aerofoil $_{\text {Crank2 }}$ (17 DVs) & & & $\sqrt{ }$ & & & $\sqrt{ }$ & $\sqrt{ }$ \\
\hline Aerofoil $_{\text {Tip }}(17 \mathrm{DVs})$ & & & & $\sqrt{ }$ & & $\sqrt{ }$ & $\sqrt{ }$ \\
\hline Wing Planform (8 DVs) & & & & & $\sqrt{ }$ & $\sqrt{ }$ & $\sqrt{ }$ \\
\hline
\end{tabular}

Note: $\mathrm{N}-\mathrm{P}_{i}$ represents $i$ th Nash-Player and P-Player indicates the Pareto-Player.

Table 4

Fitness functions for players of Hybrid-Game.

\begin{tabular}{|c|c|c|}
\hline Player & Fitness function & Optimization criteria \\
\hline Pareto-Player & $\begin{array}{l}\text { fitness }\left(f_{\text {Pareto }}\right)=\min \left(1 /\left(\overline{C_{L}}\right)\right) \\
\text { fitness }\left(f_{\text {Pareto }}\right)=\min (1 /(\overline{L / D}))\end{array}$ & $\begin{array}{l}\text { Optimize wing planform and aerofoil sections at root, crank1, crank2 and tip to maximize } \overline{C_{L}} \\
\text { and } \overline{L / D}\end{array}$ \\
\hline Nash-Player1 & fitness $\left(f_{N P 1}\right)=\min \left(1 /\left(\overline{C_{L}}\right)\right)$ & $\begin{array}{l}\text { Maximize total wing } \overline{C_{L}} \text { using design variables for Aerofoil }{ }_{\text {Root }} \text { only, all other design variables are } \\
\text { fixed }\end{array}$ \\
\hline Nash-Player2 & $\operatorname{fitness}\left(f_{N P 2}\right)=\min \left(1 /\left(\overline{C_{L}}\right)\right)$ & $\begin{array}{l}\text { Maximize total wing } \overline{C_{L}} \text { using design variables for Aerofoil }{ }_{C r a n k 1} \text { only, all other design variables } \\
\text { are fixed }\end{array}$ \\
\hline Nash-Player3 & fitness $\left(f_{N P 3}\right)=\min \left(1 /\left(\overline{C_{L}}\right)\right)$ & $\begin{array}{l}\text { Maximize total wing } \overline{C_{L}} \text { using design variables for Aerofoil }{ }_{\text {Crank2 }} \text { only, all other design variables } \\
\text { are fixed }\end{array}$ \\
\hline Nash-Player4 & fitness $\left(f_{N P 4}\right)=\min \left(1 /\left(\overline{C_{L}}\right)\right)$ & Maximize $\overline{C_{L}}$ using design variables for Aerofoil Tip $_{\text {only, other design variables are fixed }}$ \\
\hline Nash-Player5 & fitness $\left(f_{N P 5}\right)=\min (1 /(\overline{L / D}))$ & $\begin{array}{l}\text { Maximize total wing } \overline{L / D} \text { using design variables for wing planform only, all other design } \\
\text { variables are fixed }\end{array}$ \\
\hline
\end{tabular}

Note: $\overline{C_{L}}=\frac{1}{2}\left(C_{L_{\text {Sector } 2}}+C_{L_{\text {Sector4 }}}\right)$ and $\overline{L / D}=\frac{1}{2}\left(L / D_{\text {Sector2 }}+L / D_{\text {Sector } 4}\right)$.

is $M_{\infty} \in[0.7: 0.9], \alpha \in\left[6.05^{\circ}: 0.5^{\circ}\right]$ and altitude $(f t) \in[40,000: 250]$. Pareto fronts obtained by HAPMOEA and Hybrid-Game produce higher $C_{L}$ and $L / D$ when compared the baseline design. Pareto members from both optimization techniques produce similar 


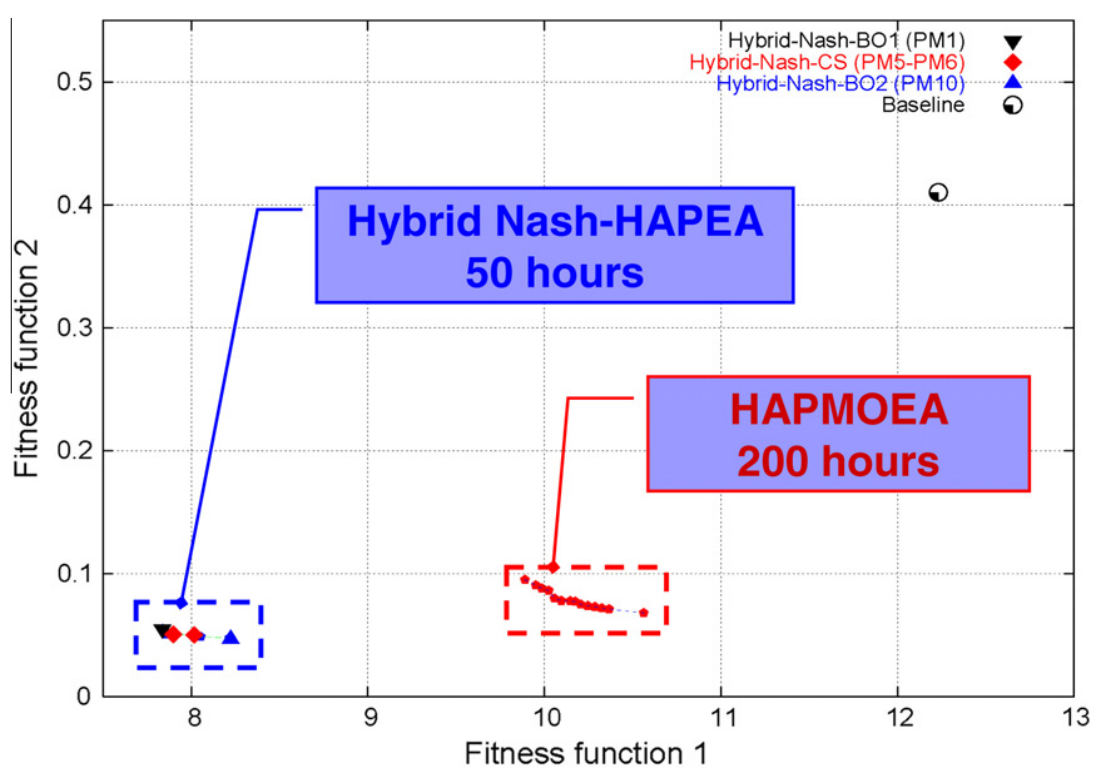

Fig. 14. Comparison of Pareto optimal front obtained by Hybrid-Game and HAPMOEA.

Table 5

Comparison of fitness values obtained by HAPMOEA and Hybrid-Game.

\begin{tabular}{|c|c|c|c|c|c|c|c|}
\hline \multirow[t]{2}{*}{ Objective } & \multirow[t]{2}{*}{ Baseline design } & \multicolumn{3}{|c|}{ HAPMOEA (200 h) } & \multicolumn{3}{|c|}{ Hybrid-Game (50 h) } \\
\hline & & PM1 (B01) & PM6 (CS) & PM15 (BO2) & PM1 (BO1) & PM6 (CS) & PM10 (BO2) \\
\hline $1 /\left(\overline{C_{L}}\right)$ & 12.232 & $9.890(-19 \%)$ & $10.096(-17 \%)$ & $10.562(-14 \%)$ & $7.836(-36 \%)$ & $8.017(-34 \%)$ & $8.223(-32 \%)$ \\
\hline $1 /(\overline{L / D})$ & 0.410 & $0.095(-77 \%)$ & $0.078(-81 \%)$ & $0.068(-83 \%)$ & $0.054(-87 \%)$ & $0.050(-88 \%)$ & $0.046(-89 \%)$ \\
\hline
\end{tabular}

Note: BO represents the best objective solution and CS stands for the compromised solution.

Table 6

Comparison of $C_{D_{\text {Quality }}}$ obtained by HAPMOEA and Hybrid-Game.

\begin{tabular}{|c|c|c|c|c|c|c|c|}
\hline \multirow[t]{2}{*}{ Objective } & \multirow[t]{2}{*}{ Baseline design } & \multicolumn{3}{|c|}{ HAPMOEA (200 h) } & \multicolumn{3}{|c|}{ Hybrid-Game $(50 \mathrm{~h})$} \\
\hline & & PM1 (B01) & PM6 (CS) & PM15 (BO2) & PM1 (BO1) & PM6 (CS) & PM10 (BO2) \\
\hline$\overline{C_{D}}$ & 12.232 & 0.025 & $0.011(-56 \%)$ & $0.009(-64 \%)$ & $0.009(-64 \%)$ & $0.009(-64 \%)$ & $0.0089(-64 \%)$ \\
\hline$\delta C_{D}$ & 0.410 & $5.49 \times 0-5$ & $1.49 \times 10^{-5}$ & $1.56 \times 10^{-5}$ & $2.11 \times 10^{-5}$ & $2.29 \times 10^{-5}$ & $2.24 \times 10^{-5}$ \\
\hline
\end{tabular}

Note: Quality is represented by mean (performance) and variance (sensitivity/stability).

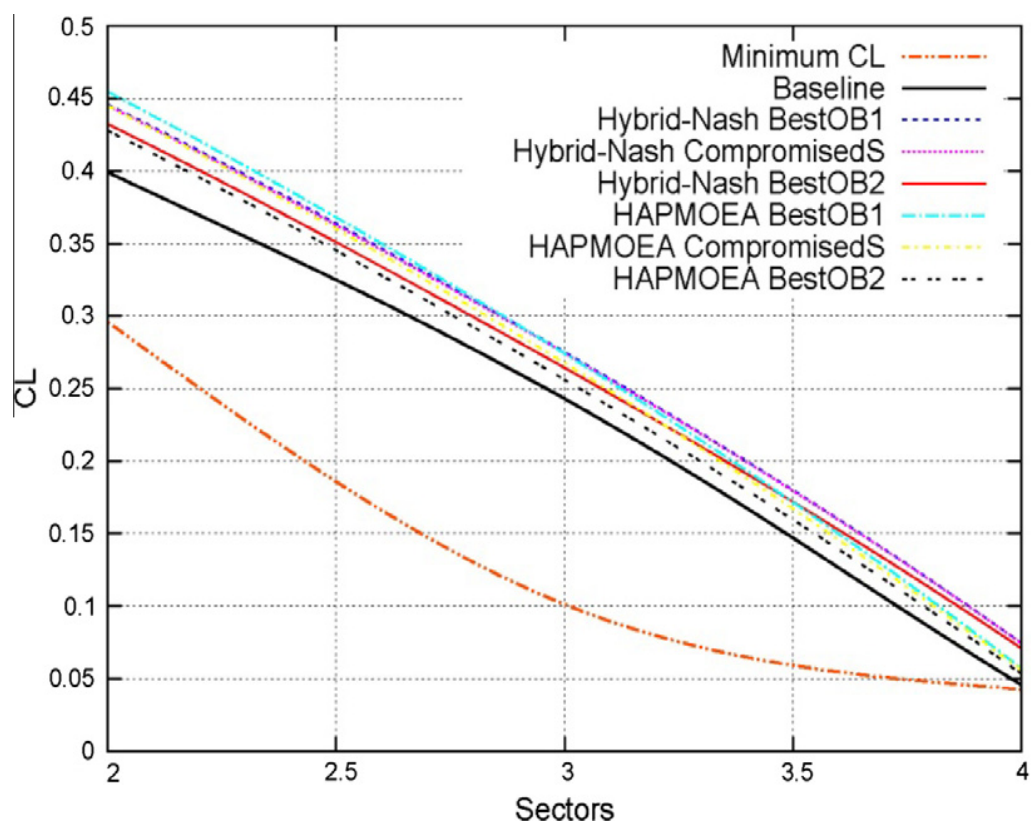

Fig. 15a. $C_{L}$ vs. Sectors: Hybrid-Nash represents Hybrid-Game. 


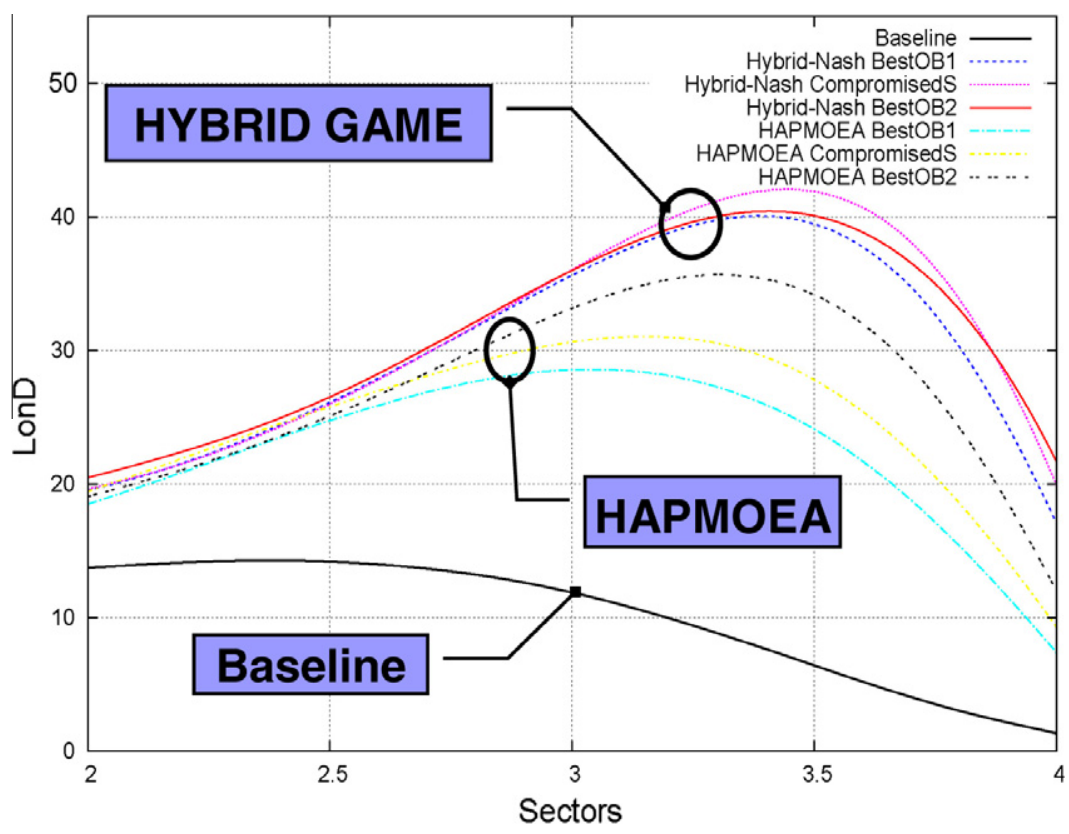

Fig. 15b. $L / D$ vs. Sectors: Hybrid-Nash represents Hybrid-Game.

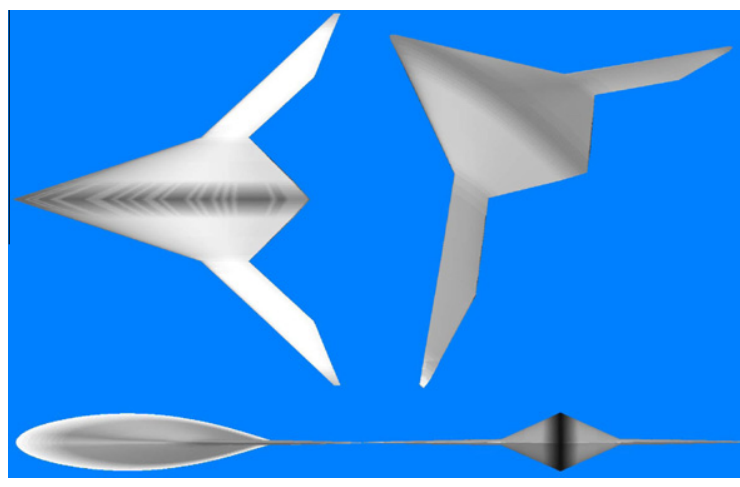

Fig. 16a. Pareto member 5 obtained by (HAPMOEA).

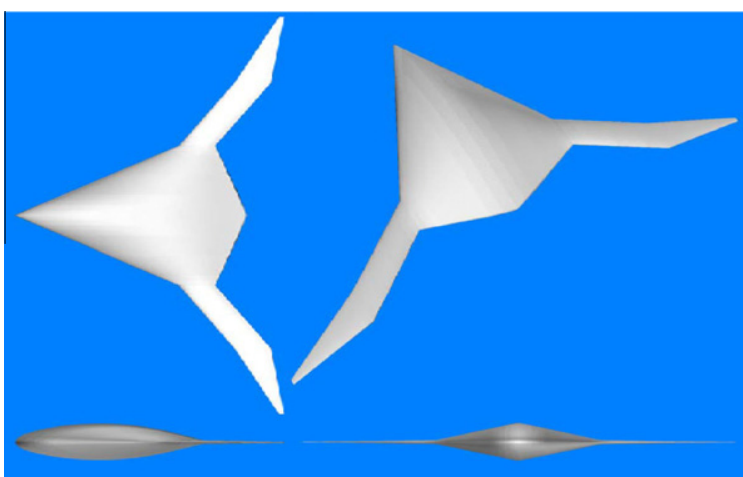

Fig. 16b. Pareto member 6 obtained by (Hybrid-Game).

results for $C_{L}$ and $L / D$ at Sector2 however the Pareto nondominated solutions from Hybrid-Game produce better $C_{L}$ and $L / D$ at Sector4.

The top, side, front and 3D view of compromised model from HAPMOEA (Pareto member 5) and Hybrid-Game (Pareto member $6)$ are shown in Figs. 16a and 16b. Even though the Hybrid-Game spent less computational time when compared to HAPMOEA, both compromised solutions are geometrically similar.

\subsection{Summary}

This paper explored the optimization methods: HAPMOEA and Hybrid-Game (Hybridised MOEA) for solving multi-objective design optimization UCAV. HAPMOEA has been hybridised by using Nash-Game without hierarchical multi-population/fidelity topology: Hybrid-Game. Numerical results show that by introducing Nash-Game as a companion optimizer to help or guide the multiobjective evolutionary optimizer to capture a fast Pareto non-dominated front. It is also shown that the Hybrid-Game (Hybridised MOEA) reduces the computational cost while generating better Pareto front when compared to HAPMOEA. The reason why Hybrid-Game has superiority in terms of optimization efficiency is that the Nash-Game decomposes one complex multi-objective design problem into five simpler single-objective design problems corresponds to five Nash-Players.

\section{Conclusions}

The optimization methods HAPMOEA and Hybrid-Game were demonstrated and they were implemented to solve multi-objective design problem. It is shown that both methods produce a set of useful Pareto non-dominated solutions. It was also shown that the coupling of Pareto optimality and Nash-Game; Hybrid-Game has superiority on both computational efficiency and solution quality when compared to HAPMOEA. Both methodologies couple a robust MOEA, with aerodynamic analysis tools. A family of Pareto optimal designs obtained by both HAPMOEA and Hybrid-Game provides a selection to the design engineers to proceed into more detail phases of the design process. Even though the numerical results of the methods show the simultaneous improvement in UCAV aerodynamic performance on $C_{L}$ and $L / D$ there is a fluctuation between Sector2-Sector3 and Sector3-Sector4 which can cause flight control or structural failures. This fluctuation can be avoided by using uncertainty design technique during optimization. Future work will focus on coupling Hybrid-Game and high fidelity aerody- 
namics and electromagnetic analysis tools under uncertainty in flight conditions and electromagnetic frequencies.

\section{Acknowledgements}

The authors gratefully acknowledge E.J. Whitney and M. Sefrioui Dessault Aviation for discussions on Hierarchical EAs. We would like to acknowledge A. Jameson and David A. Caughy for granting the potential flow software, and W.H. Mason for the use of FRICTION.

\section{References}

[1] Tang Z, Périaux J, Désidéri J-A. Multi criteria robust design using adjoint methods and game strategies for solving drag optimization problems with uncertainties. In: East west high speed flow fields conference 2005, Beijing. China, 19-22 October, 2005. p. 487-93.

[2] Taguchi G, Chowdhury S. Robust engineering. New York: McGraw-Hill; 2000.

3] Lee DS, Gonzalez LF, Periaux J, Srinivas K. Evolutionary optimisation methods with uncertainty for modern multidisciplinary design in aeronautical engineering, Notes on numerical fluid mechanics and multidisciplinary design (NNFM 100), 100 volumes NNFM and 40 years numerical fluid mechanics. Heidelberg: Springer-Berlin; 2009. p. 271-84. ISBN 978-3-54070804-9 [chapter 3.]

[4] Periaux J, Mantel B, Chen HQ. Genetic algorithms applied to domain decomposed flow computations. In: D'esid'eri R-A, Hirsch C, Tallec P, Pandolfi M, Periaux J, editors. Computational fluid dynamics '96 proc third ECCOMAS computational fluid dynamics conference, Paris France, 1996. p. 866-70.

[5] Periaux J, Chen HQ Mantel B, Sefrioui M, Sui HT. Combining game theory and genetic algorithms with application to DDM-nozzle optimization problems. Finite Elem Anal Des 2001;5(5):417-29. ISSN:0168-874X.

[6] Lee DS, Gonzalez LF, Whitney EJ. Multi-objective, multidisciplinary multifidelity design tool: HAPMOEA - user guide; 2007.

[7] Sefrioui M, Periaux J. Nash genetic algorithms: examples and applications. In: Proceedings of the 2000 congress on evolutionary computation CEC00. IEEE Press, La Jolla Marriott Hotel La Jolla, California, USA, 2000. p. 509-16. ISBN:07803-6375-2.

[8] Deb K. Multi-objective evolutionary algorithms: introducing bias among pareto-optimal solutions. KanGAL report no. 99002. Kanpur Genetic Algorithms Laboratory, Department of Mechanical Engineering, Indian Institute of Technology Kanpur, Kanpur 208016, India, 1999.

[9] Lee DS. Uncertainty based multiobjective and multidisciplinary design optimization in aerospace engineering, The Univ. of Sydney, Sydney, NSW, Australia, section 10.7, 2008. p. 348-70.
10] Koza J. Genetic programming II. Massachusetts Institute of Technology, 1994

[11] Michalewicz Z. Genetic algorithms + data structures = evolution programs. Artificial intelligence. Springer-Verlag; 1992.

[12] Hansen N, Ostermeier A. Completely derandomized self-adaptation in evolution strategies. Evolut Comput 2001;9(2):159-95.

[13] Hansen N, Müller SD, Koumoutsakos P. Reducing the time complexity of the derandomized evolution strategy with covariance matrix adaptation (CMAES). Evolut Comput 2003;11(1):1-18.

[14] Wakunda J, Zell A. Median-selection for parallel steady-state evolution strategies. In: Schoenauer Marc, Deb Kalyanmoy, Rudolph Günter, Yao Xin, Lutton Evelyne, Julian Juan Merelo, editors. Parallel problem solving from nature - PPSN VI. Berlin; Springer; 2000. p. 405-14.

[15] Van Veldhuizen DA, Zydallis JB, Lamont GB. Considerations in engineering parallel multiobjective evolutionary algorithms. IEEE Trans Evolut Comput 2003;7(2):144-73.

[16] Sefrioui M, Périaux J. A hierarchical genetic algorithm using multiple models for optimization. In: Schoenauer M, Deb K, Rudolph G, Yao X, Lutton E, Merelo JJ, et al., editors. Parallel problem solving from nature, PPSN VI. Springer; 2000. p. 879-88.

[17] Lee DS, Gonzalez LF, Periaux J. UAS Mission Path Planning System (MPPS) Using hybrid-game coupled to multi-objective design optimizer. J Dyn Syst- TASME 2010; 132(4):041005-1-11. ISSN 00220434.

[18] Deb K, Agrawal S, Pratap A, Meyarivan T. A fast and elitist multi-objective genetic algorithm: NSGA-II. IEEE Trans Evolut Comput 2002;6(2):182-97.

[19] Lee DS, Gonzalez LF, Periaux J, Srinivas K. Hybrid-game strategies coupled to evolutionary algorithms for robust multidisciplinary design optimization in aerospace engineering. IEEE Trans Evolut Comput, in press, TEVC-00213-2009.

[20] Lee DS, Gonzalez LF, Srinivas K, Periaux J. Robust evolutionary algorithms for UAV/UCAV aerodynamic and RCS design optimisation. Int J Comput Fluids 2008;37(5):547-64. ISSN: 0045-7930.

[21] Lee DS, Gonzalez LF, Srinivas K, Periaux J. Robust design optimisation using multi-objective evolutionary algorithms. Int J Comput Fluids 2008;37(5):565-83. ISSN:0045-7930.

[22] Deb K. Multi-objective optimization using evolutionary algorithms. Chichester (UK): Wiley; 2001

[23] Deb K. Nonlinear goal programming using multi-objective genetic algorithms J Operat Res Soc 2001;52(3):291-302.

[24] Jameson A. Caughey DA, Newman PA, Davis RM. NYU transonic swept-wing computer program - FLO22, Langley Research Center, 1975.

[25] Mason W. Applied computational aerodynamics. Appendix D: programs, Tuesday, January 21, 1997.

[26] Geer H, Bolkcom C. Unmanned aerial vehicles: background and issues for congress. CRC report for congress, 2005.

[27] Francis MS. AUVSI's unmanned systems program review. Association for Unmanned Vehicle Systems International, Washington, DC, 2005.

[28] Northrop Grumman. <http://www.is.northropgrumman.com/systems/ nucasx47b.html>. 